\title{
ANALYSIS AND DESIGN OF REINFORCED CONCRETE STEPPED CANTILEVER RETAINING WALL
}

\author{
S.S Patil ${ }^{1}$, A.A.R.Bagban ${ }^{2}$ \\ ${ }^{1}$ Professor and Head, Civil Engineering Department, Walchand Insitute of Technology, Solapur, Maharashtra, India \\ ${ }^{2}$ Post Graduate Student, Walchand Institute of Technology, Solapur, Maharashtra, India
}

\begin{abstract}
A retaining wall is one of the most important types of retaining structures. It is extensively used in variety of situations such as highway engineering, railway engineering, bridge engineering and irrigation engineering. Reinforced concrete retaining walls have a vertical or inclined stem cast with base slab. These are considered suitable up to a height of 6m.It resists lateral earth pressure by cantilever action of stem, toe slab and heel slab. The tendency of wall to slide forward due to lateral earth pressure should be investigated and a factor of safety of 1.5 shall be provided against sliding. Cantilever retaining walls are found best up to a height of 6m.For greater heights earth pressure due to retained fill will be higher due to lever arm effect, higher moments are produced at base, which leads to higher section for stability design as well as structural design. This proves to be an uneconomical design. As an alternative to this, one may go for counter fort retaining wall, which demands greater base area as well as steel. As a solution to this difficulty, a new approach that is to minimize effect of forces coming from retained fill, short reinforced concrete members in the form of cantilever steps are cast along the stem on the retaining face. Addition of these steps would counterbalance the locally appearing forces and will result into lesser moment and shear forces along the stem. Also it will reduce the bending action that is pressure below the base.
\end{abstract}

The objectives of the study are

1) To reduce the stresses on the retaining face of the cantilever retaining wall, it is proposed to introduce reinforced concrete steps along the stem.

2) Decide the most economical location of step along length and also along height of wall from number of trials.

3) Decide cross section of the R. C. step as per the stresses due to frictional forces in step.

4) Stability analysis of Cantilever retaining wall with steps for unit width will be done. Check for minimum and maximum stresses will be observed.

5) Cost comparison shall be carried out for these three different alternatives to give most economical retaining wall type.

Keywords: Mechanism of step, Finalization of Step location, Stabilizing frictional force, Concrete quantity, Steel reinforcement and Cost Comparison of Counter fort and Stepped Cantilever retaining wall.

$$
\text { - ****. }
$$

\section{INTRODUCTION}

A retaining wall is one of the most important types of soil retaining structures. The primary purpose of retaining wall is to retain earth or other material at or near vertical position. It is extensively used in variety of situations such as highway engineering, railway engineering, bridge engineering, dock and harbor engineering, irrigation engineering, land reclamation and coastal engineering etc. Reinforced concrete retaining walls have a vertical or inclined stem cast monolithic with a base slab. These are considered suitable up to a height of $6 \mathrm{~m}$. It resists the lateral earth pressure by cantilever action of the stem, toe slab and heel slab. Necessary reinforcements are provided to take care of the flexural stresses. The tendency of the wall to slide forward due to lateral earth pressure should be investigated and if a factor of safety is insufficient, a shear key should be designed to prevent lateral movement of the structure.

\subsection{Cantilever Retaining Walls}

These walls are made of reinforced cement concrete. It consists of a thin stem and a base slab cast monolithically. This type of wall is found to be economical up to a height 6 to $8 \mathrm{~m}$.

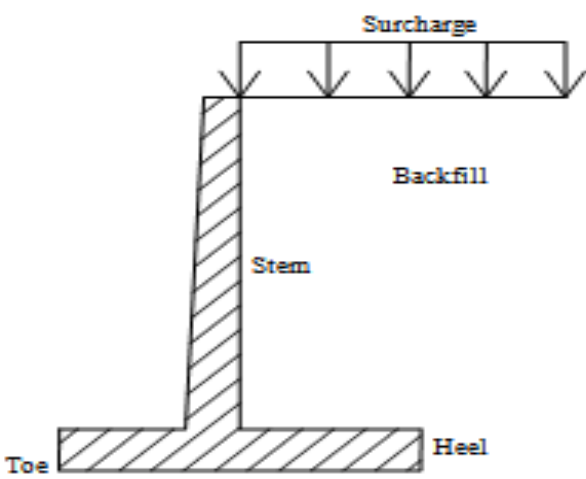

Fig - 1 


\subsection{Counter fort Retaining Walls}

These walls have thin vertical slabs, known as counter forts, spaced across vertical stem at regular intervals. The counter forts tie the vertical stem with the base slab. Thus the vertical stem and the base slab span between the counter forts. The purpose of providing the counter forts is to reduce the shear force and bending moments in the vertical stem and the base slab. The counter fort retaining walls are economical for a height more than 6 to $8 \mathrm{~m}$.

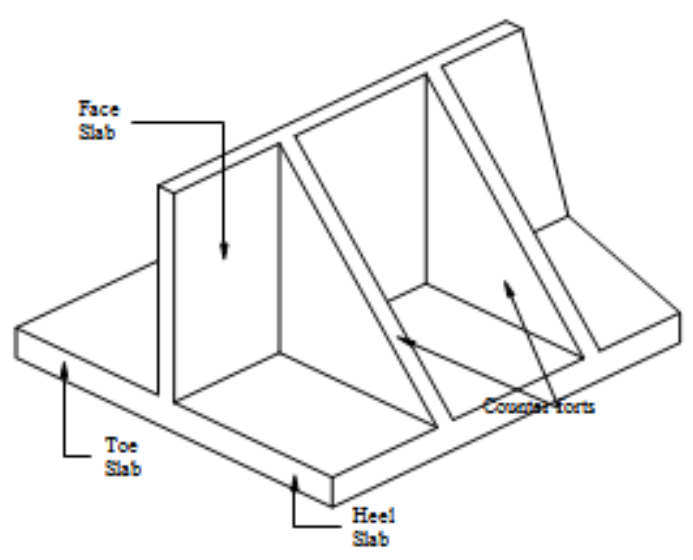

Fig - 2

\section{ANALYSIS OF RETAINING WALLS}

\subsection{Cantilever retaining wall}

\section{A) Stability:}

Figure 3 shows a cantilever retaining wall subjected to following forces:

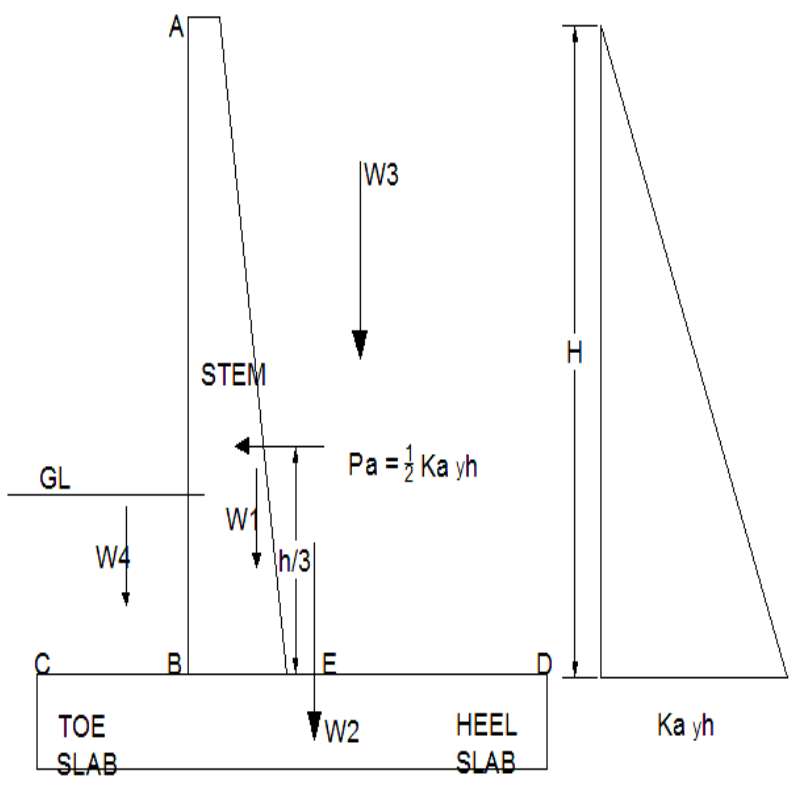

Fig - 3: Mechanism of Cantilever Retaining Wall
- Weight W1 of the stem

- Weight W2 of the base slab

- Weight W3 of the column of soil supported on heel slab

- Weight W4 of the soil supported on toe slab

- Horizontal force Pa equal to active earth pressure acting at $\mathrm{H} / 3$ above base slab.

\section{B) Modes of Failure of a Retaining Wall:}

\section{- Overturning about $\mathrm{A}$}

The most hazardous mode of failure of retaining wall is due to overturning because of unbalanced moments. Here, a minimum factor of safety is used.

- Sliding:

The horizontal force tends to slide the stem and wall away from fill. The tendency to resist this is achieved by the friction at the base. Here, if the wall is found to unsafe against sliding, shear key below the base is provided. Such a key develops passive pressure which resists completely the sliding tendency of wall. A factor of safety of 1.5 is used against sliding.

\section{- Bending Failure}

The stem $\mathrm{AB}$ will bend as cantilever so that tensile face will be towards the soil face in case if there is no backfill, where as tensile face will be towards the water face in case there is backfill. The critical section will be at $\mathrm{E}$ and $\mathrm{B}$, where crack may occur at if it is not properly reinforced. The soil side slab will have net pressure acting downwards, and will bent as a cantilever having tensile face at top for retaining wall, at the same time the heel slab will be subjected to net upward pressure causing tensile face at bottom. The thickness of stem, toe slab, and heel slab must be sufficient to withstand compressive stresses due to bending also; the stem thickness must be check for uncracked section.

\subsection{Design Principal of Cantilever Retaining Wall}

The various dimensions of wall are so proportioned that the various failure criteria discussed above are taken care of. The design of wall consist of the fixation of base width, design of stem, design of toe slab, design of heel slab.

\section{A) Fixation of Base Width:}

The base width of wall is so chosen that the resultant of forces remain within middle third of base slab, the uplift pressure is zero at heel slab side also it should be safe from consideration of sliding.

\section{B) Design of Stem:}

The vertical stem is designed as cantilever for triangular loading with Kayh as base of triangle $h$ as height of it. The main reinforcement is provided at $0.3 \%$ of the area of cross section along the length of wall. 


\section{C) Design of Toe Slab:}

It is also design as a cantilever beam or slab. The main reinforcement is provided at lower face or bottom side as upward soil pressure load is acting on that face. Thickness is checked for maximum cantilever moment and deflection criterion.

\section{D) Design of Heel Slab:}

It is also design as a cantilever beam or slab. The main reinforcement is provided at the upper face or top side of heel slab as active load is acting there in form of overburden pressure. The design reinforcement for effective moment due to upward soil pressure should also provide at bottom side of heel slab. The thickness is checked for maximum cantilever moment and deflection criterion for cantilever action.

\subsection{Analysis of Counter-Fort Retaining Wall:}

The counter forts support both the vertical stem as well as base slab. Design principles for various component parts are discussed below in brief. The same criterion is adopted for fixing the base width as cantilever retaining wall.

\section{A) Design of stem}

Unlike the stem of cantilever retaining wall, the stem of counter fort retaining wall acts as a continuous slab supported on counters forts. Due to varying pressure over the height of stern, the stem slab deflects outwards and hence main reinforcement is provided along the length of the wall as per design conditions.

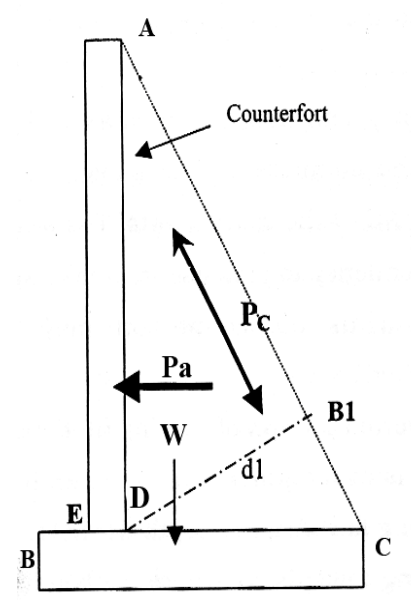

Section

Pc - Effect of counter fort

Lc - Spacing of counter forts along length of wall

Fig - 4: Mechanism of Counter fort Retaining Wall

The reaction of the stem is taken by the counter forts, to which it is firmly anchored. The maximum bending moment occurs at Base. The uniformly distributed earth pressure load or water pressure load is calculated for unit height.

\section{B) Design of Heel Slab}

The action is similar that of stem. The Heel slab is subjected to the downward load due to weight of soil and self weight, upward load due to upward soil pressure below heel slab. The maximum net pressure is found to act on a strip of unit width near outer edge, since the upward soil reaction is minimum there, the total reaction from the heel slab is transferred to the counter forts, and this load helps to provide a balancing moment against its overturning. The heel slab is firmly attached to the counter forts by means of vertical ties.

\section{C) Design of Toe Slab}

The action of Heel slab is similar to that of cantilever retaining wall.

\section{D) Design of Counter Forts}

The counter fort takes reactions, both from the stem as well as Heel slab. As shown in fig. 4.2, the counter forts are subjected to tensile stresses along the outer face AC of the counter forts. The angle $\mathrm{ABC}$ between stem and slab has a tendency to increase from 900, and counter forts resist this tendency. Thus the counter fort may be considered to bend as a cantilever, fixed at BC. The counter fort acts as an inverted $\mathrm{T}$ beam of varying rib depth. The maximum depth of this $\mathrm{T}$ beam is at the junction $\mathrm{B}$. The depth is measured perpendicular to the sloping face $A B$, i.e. depth $d l=B B 1$. At $\mathrm{B}$, This depth thus goes on decreasing towards $\mathrm{Al}$ where the bending moment also decreases. The width of counter fort is kept constant throughout its height; Main reinforcement is provided parallel to $\mathrm{AC}$ The faces $\mathrm{AB}$ and $\mathrm{BC}$ of the counter fort remain in compression. The compressive stresses on face $A B$ are counterbalanced by the vertical upward reaction transferred by the slab. In addition to the main reinforcement, the counter forts are jointed firmly to the stem and base slab by horizontal and vertical ties respectively.

\section{4: Stepped Cantilever Retaining Wall (New Approach)}

For retaining back fill of heights more than 10-11 meters. The conventional walls like cantilever and counter fort becomes very massive and almost uneconomical hence a suitable modification to these walls so as to economize the retaining wall construction. The proposed modified alternative is 'Stepped cantilever retaining wall'. The general outline of concept will be clear from figure as shown. 

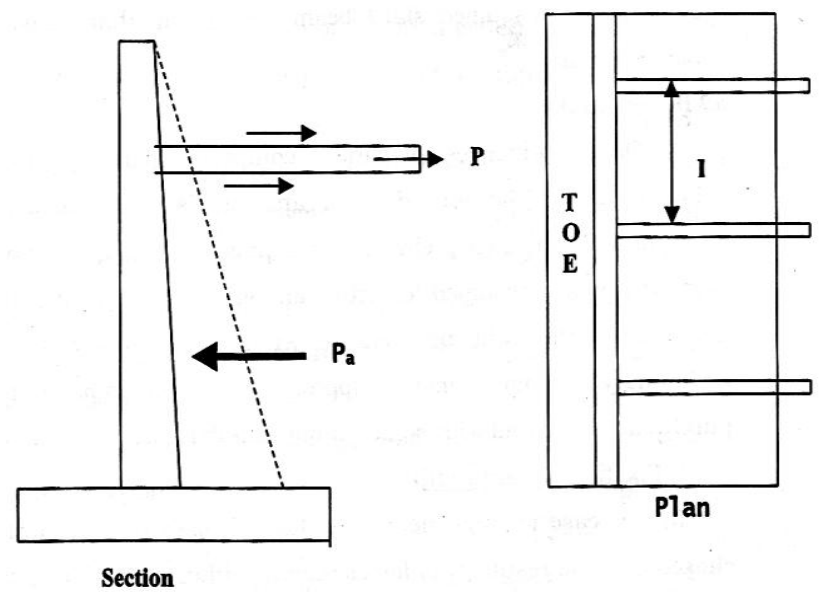

P - Stabilizing frictional force

$\mathrm{Pa}$ - Active pressure component

L - Spacing of concrete steps along length of wall

Fig - 5: Mechanism of stepped cantilever retaining wall

The main concept in this type is supporting the high stem at critical points indirectly by means of pulling force developed due to surface Friction of concrete steps with backfill. Here the effect of self weight of these steps in stabilizing wall against active pressure is not considered as it may be negligible. Conventionally in case of sheet pile walls, there was use of anchor rods and the concrete plates or concrete dead man was used to develop frictional force. In case of sheet pile wall with vertical concrete plates the mechanism of pulling force was due to passive resistance of soil mass bounded by height of concrete wall and in that case the role of concrete wall was different from frictional resistance function. In case of sheet pile walls the thickness of stem was very small but it is continuous wall with membrane action than beam/slab action but in this case, these concrete steps are used as supporting mechanism for conventional cantilever wall which gives relatively less dimensions for assumed slab beam mechanism than conventional design approach.

\section{A) Design Principles}

Design principles for various component parts are discussed below in brief. The procedure of analysis is same as cantilever retaining wall but their preliminary dimensions given will be based on load distribution assumed for actual analysis. Like any other analysis and design this will be Iterative (trial and error) method, the preliminary dimensions may be approximately given as half of that for purely cantilever wall with some exiting thumb rules.

\section{B) Fixation of Base Width}

In this case it is not necessary that the base width of wall is so chosen that the resultant of forces remain within middle third and the minimum (uplift) pressure at toe is zero but these dimensions can be chosen approximately without these checks.

\section{C) Design of Stem}

The vertical stem is designed as cantilever for triangular loading but reinforcement will be provided from actual modified Pressure diagram due to restoring force developed by concrete steps. Distribution reinforcement may be provided as per standards.

\section{D) Design of Toe Slab}

It is also designed as a cantilever slab/beam. Reinforcement is provided at lower face. There will major reduction in depth and steel reinforcement in toe and heel slab due to reduction in the active pressure and addition in self-weight of wall. This will effectively economize the wall construction. Thickness is checked for the maximum cantilever moment.

\section{E) Design of Heel Slab}

It is also designed as a cantilever. Reinforcement is provided at the upper face. Thickness is checked for the maximum cantilever moment.

\section{F) Design of Concrete Steps}

The concrete steps will be placed along length at suitable spacing L. The mechanism of friction generation is fully dependant on overburden load i.e. depth of step from top of wall hence the step provided at more depth will give better results. The one more effective element in friction development is embedment length and width of step. The overlaying or overlapping of steps and embedment in various pressure zones like passive or rest will also be important. These steps will act as free cantilevers spanning from stem or somewhat like plates supported on spring or elastic media depending upon degree of compaction of backfill. These assumptions dominate its design or depth at stem and free end. If steps assumed as slab strips supported on elastic media then their depth and steel reinforcement for moment will be less than its minimum depth as per standards and steel required for tensile forces developed due to frictional resistance.

\section{G) Calculation of Frictional Resistance offered by}

\section{Plate}

The concrete plates are inserted in compacted backfill. They will develop frictional force along contact planes of concrete and soil due to overburden pressure and compaction. This frictional force will act as indirect stabilizing force for overturning retaining wall and will pull wall inside. 


\section{Mechanism}

The concrete plate separated from stem inserted in soil is as shown in figure 4.

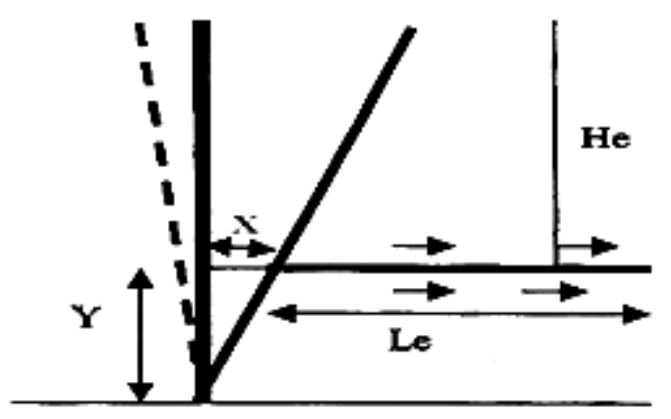

Displaced stem

Active state zone

Fig - 6: Mechanism of step

- The effective frictional pressure=Coefficient of friction $\mathrm{x}$ height of backfill on plate $\mathrm{x}$ dry density of backfill.

\section{1) Stepped Retaining Wall of Height $6 \mathrm{~m}$ :}

\section{Assumptions}

1. Back fill is enough compacted.

2. Step length embedded in backfill $-3.5 \mathrm{~m}$

3. Step dimensions $-400 \times 300 \mathrm{~mm}$
- $\quad$ Effective length of plate =Length of plate beyond active zone.

- $\quad$ Effective frictional force $=$ Width of plate $\mathrm{x}$ effective length of plate $\times 2$ x Effective frictional pressure.

\section{H) Finalization of Step Location}

For actual analysis to decide location of step along length and along height of wall is most important task as it may hamper most of assumptions. Hence the length of step immersed in backfill was kept constant and the location of plate along length of wall was fixed from number of trails for stability. For finalizing the location of step along height of wall, the number of trials is taken starting from half of height and with interval of $500 \mathrm{~mm}$. The stability analysis of each wall is done and concrete quantity, steel reinforcement and cost per meter are compared. The most economical wall is selected for final comparison as alternative with other retaining wall types. The following table shows all aspects of stepped cantilever wall for various step heights from top of wall. The comparison is also shown graphically by subsequent graphs for each height.

Table 1: Stability analysis and cost comparison

\begin{tabular}{|l|l|l|l|l|l|l|}
\hline $\begin{array}{l}\text { Step from } \\
\text { top m. }\end{array}$ & $\begin{array}{l}\text { Width of } \\
\text { toe slab }\end{array}$ & $\begin{array}{l}\text { Width of } \\
\text { heel slab }\end{array}$ & $\begin{array}{l}\text { Depth of } \\
\text { base slab }\end{array}$ & $\begin{array}{l}\text { Total base } \\
\text { slab }\end{array}$ & $\begin{array}{l}\text { Stem thick } \\
\text { m }\end{array}$ \\
\hline & & & & & At top & Bottom \\
\hline 3 & 0.85 & 2.5 & 0.4 & 3.7 & 0.2 & 0.35 \\
\hline 3.5 & 0.65 & 2.5 & 0.4 & 3.5 & 0.2 & 0.35 \\
\hline $\mathbf{4}$ & $\mathbf{0 . 6 5}$ & $\mathbf{1 . 9}$ & $\mathbf{0 . 4}$ & $\mathbf{2 . 9}$ & $\mathbf{0 . 2}$ & $\mathbf{0 . 3 5}$ \\
\hline 4.5 & 0.65 & 2.42 & 0.4 & 3.42 & 0.2 & 0.35 \\
\hline 5 & 0.65 & 2.6 & 0.4 & 3.6 & 0.2 & 0.35 \\
\hline 5.5 & 0.65 & 2.9 & 0.45 & 3.9 & 0.2 & 0.35 \\
\hline
\end{tabular}

\begin{tabular}{|l|l|l|l|l|l|}
\hline $\begin{array}{l}\text { Upward } \\
\text { pressure } \\
\text { KN/m2 }\end{array}$ & $\begin{array}{l}\text { Soil } \\
\text { in }\end{array}$ & $\begin{array}{l}\text { Effective } \\
\text { frictional force }\end{array}$ & $\begin{array}{l}\text { Concrete } \\
\mathrm{m} 3\end{array}$ & $\begin{array}{l}\text { Steel quantity } \\
\text { Kg/m }\end{array}$ & Cost Rs/m \\
\hline Pmax. & Pmin. & & & & \\
\hline 101.7 & 295.2 & 38.21 & 2.305 & 138.26 & 14012.68 \\
\hline 103.6 & 292 & 51.85 & 2.3625 & 143.3 & 14430.65 \\
\hline $\mathbf{8 8}$ & $\mathbf{2 9 6 . 5}$ & $\mathbf{6 7 . 5 6}$ & $\mathbf{2 . 2 6}$ & $\mathbf{1 4 1 . 3 8}$ & $\mathbf{1 3 9 8 9 . 3 4}$ \\
\hline 94.7 & 299.1 & 85.36 & 2.6055 & 158.99 & 15955.82 \\
\hline 101.7 & 296.1 & 105.23 & 2.815 & 176.65 & 17448.45 \\
\hline 106.9 & 305.4 & 127.18 & 3.2675 & 202.55 & 20145.9 \\
\hline
\end{tabular}




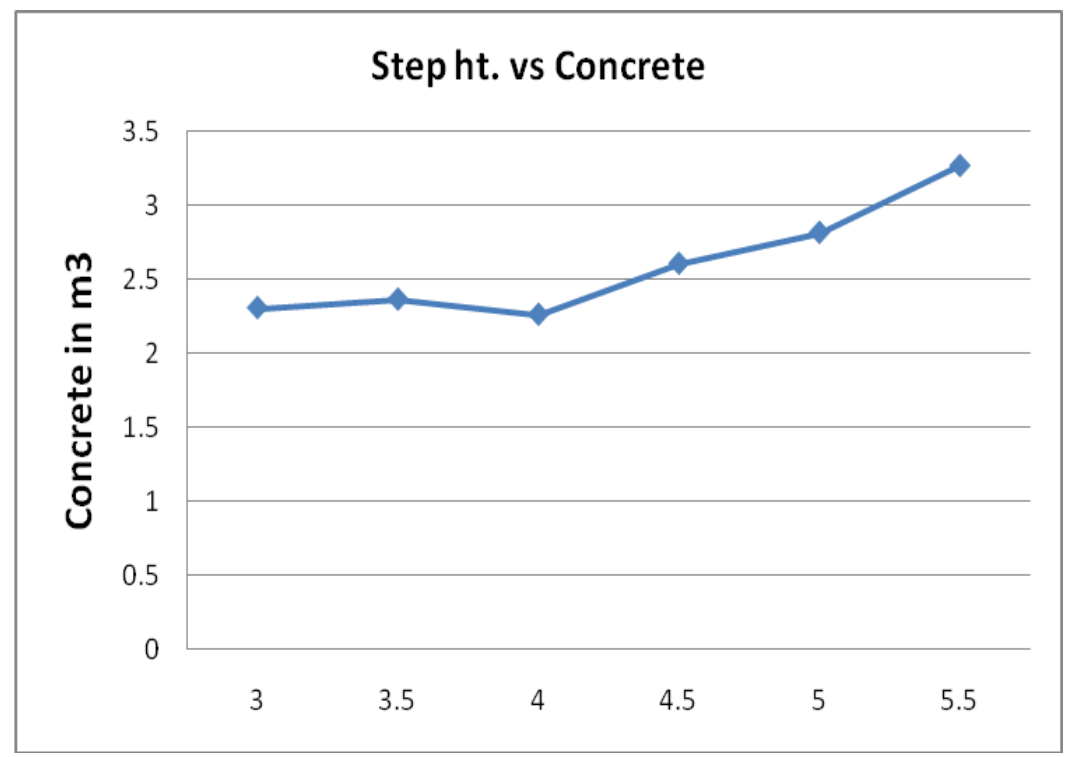

Graph 1: Step location Vs concrete $\mathrm{m} 3$ for wall Ht. $6.0 \mathrm{~m}$

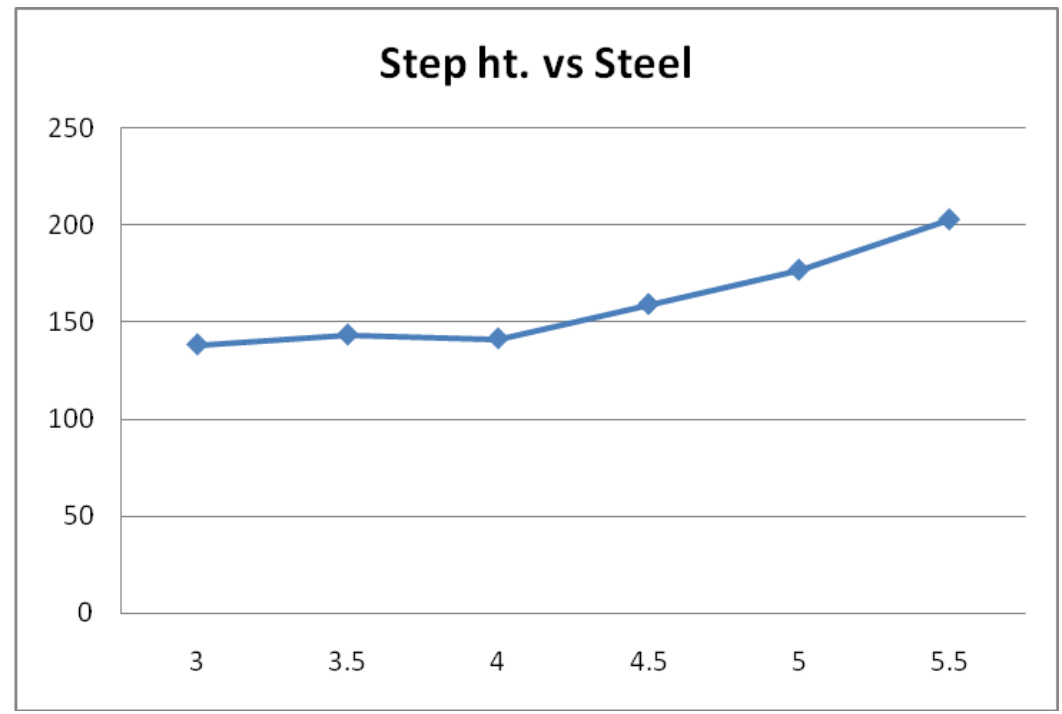

Graph 2: Step location Vs steel kg for wall Ht. 6m

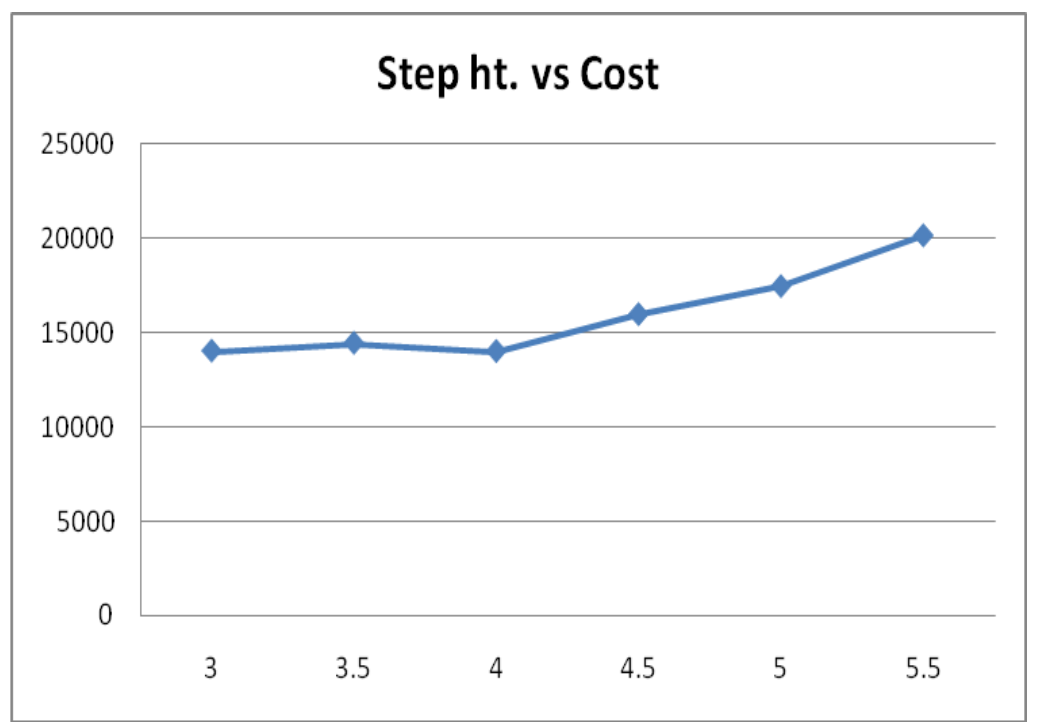

Graph 3: Step location Vs cost per meter for wall Ht. $6.0 \mathrm{~m}$ 


\section{2) Stepped Retaining Wall of Height 8m:}

\section{Assumptions}

1. Back fill is enough compacted.

2. Step length embedded in backfill $-4.5 \mathrm{~m}$

3. Step dimensions - $500 \times 300 \mathrm{~mm}$

Table2: Stability analysis and cost comparison for wall ht. $8 \mathrm{~m}$

\begin{tabular}{|l|l|l|l|l|l|l|}
\hline $\begin{array}{l}\text { Step from } \\
\text { top }\end{array}$ & $\begin{array}{l}\text { Width of } \\
\text { toe slab }\end{array}$ & $\begin{array}{l}\text { Width of } \\
\text { heel slab }\end{array}$ & $\begin{array}{l}\text { Depth of } \\
\text { base slab }\end{array}$ & $\begin{array}{l}\text { Total base } \\
\text { slab }\end{array}$ & $\begin{array}{l}\text { Stem thickness } \\
\text { in m }\end{array}$ & Bottom \\
\hline & & & & at top & \\
\hline 4 & 1.3 & 3.95 & 0.47 & 5.65 & 0.25 & 0.4 \\
\hline 4.5 & 1.2 & 3.9 & 0.5 & 5.5 & 0.25 & 0.4 \\
\hline 5 & 1.1 & 3.85 & 0.5 & 5.35 & 0.25 & 0.4 \\
\hline $\mathbf{5 . 5}$ & $\mathbf{0 . 9 5}$ & $\mathbf{3 . 9}$ & $\mathbf{0 . 4 5}$ & $\mathbf{5 . 2 5}$ & $\mathbf{0 . 2 5}$ & $\mathbf{0 . 4}$ \\
\hline 6 & 1.15 & 3.4 & 0.45 & 5 & 0.25 & 0.45 \\
\hline 6.5 & 1.25 & 3.2 & 0.45 & 4.9 & 0.25 & 0.45 \\
\hline 7 & 1.35 & 3.12 & 0.45 & 4.995 & 0.25 & 0.525 \\
\hline 7.5 & 1.45 & 3.15 & 0.45 & 5.2 & 0.25 & 0.6 \\
\hline
\end{tabular}

\begin{tabular}{|l|l|l|l|l|l|}
\hline $\begin{array}{l}\text { Upward soil } \\
\text { pressure } \\
\text { KN/m2 }\end{array}$ & $\begin{array}{l}\text { Effective } \\
\text { frictional } \\
\text { force KN }\end{array}$ & $\begin{array}{l}\text { Concrete } \\
\mathrm{m} 3\end{array}$ & $\begin{array}{l}\text { Steel } \\
\text { quantity } \\
\mathrm{Kg} / \mathrm{m}\end{array}$ & Cost Rs/m \\
\hline Pmax. & Pmin. & & & & \\
\hline 220.63 & 281.53 & 78.91 & 3.9555 & 294.49 & 26507.32 \\
\hline 213.15 & 299.95 & 100.46 & 4.2125 & 272.96 & 26481.03 \\
\hline 217.81 & 295.3 & 124.61 & 4.3 & 280.63 & 27117.09 \\
\hline 206.31 & 294.54 & 151.35 & 4.15 & 275.03 & 26351.29 \\
\hline 197.56 & 295.12 & 180.68 & 4.35 & 320.84 & 29021.12 \\
\hline 183.01 & 295.3 & 212.62 & 4.48 & 313.44 & 29157.92 \\
\hline 177.32 & 289.68 & 247.15 & 4.96025 & 295.81 & 30080.705 \\
\hline 172.75 & 286.56 & 284.28 & 5.5275 & 308.79 & 32624.22 \\
\hline
\end{tabular}

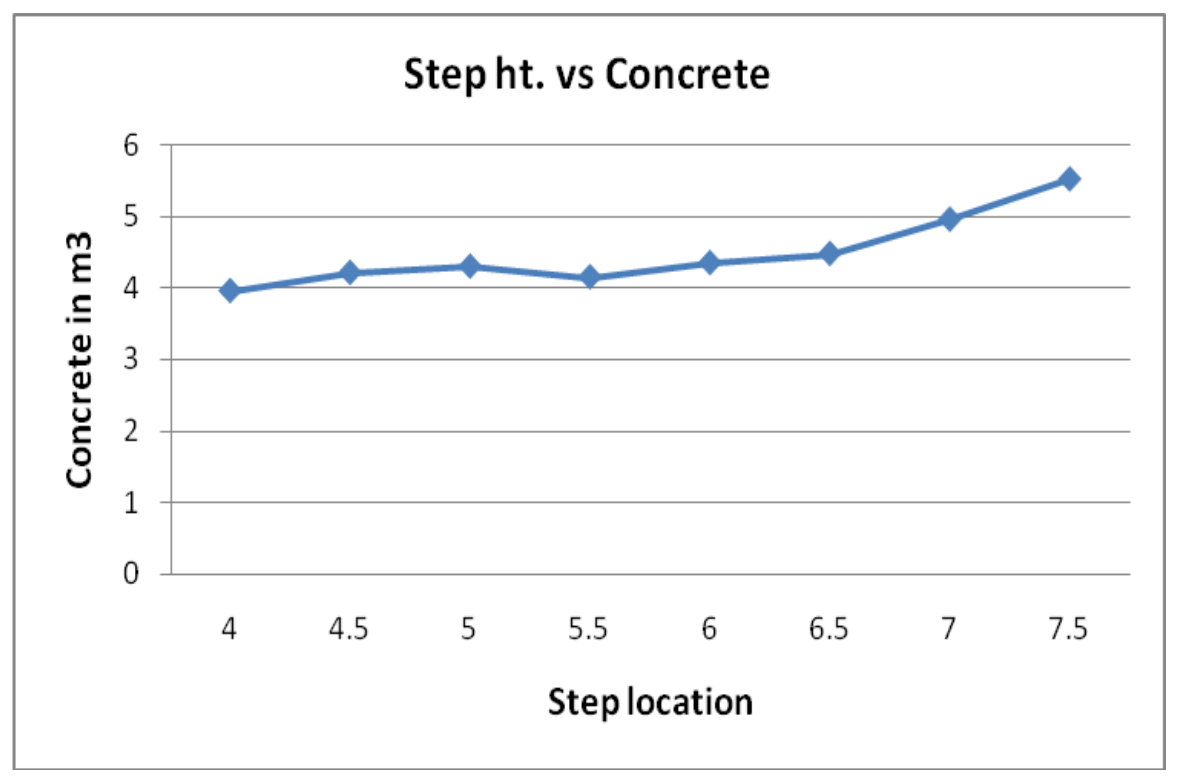

Graph 3: Step location Vs concrete cum/m for wall Ht. $8.0 \mathrm{~m}$ 


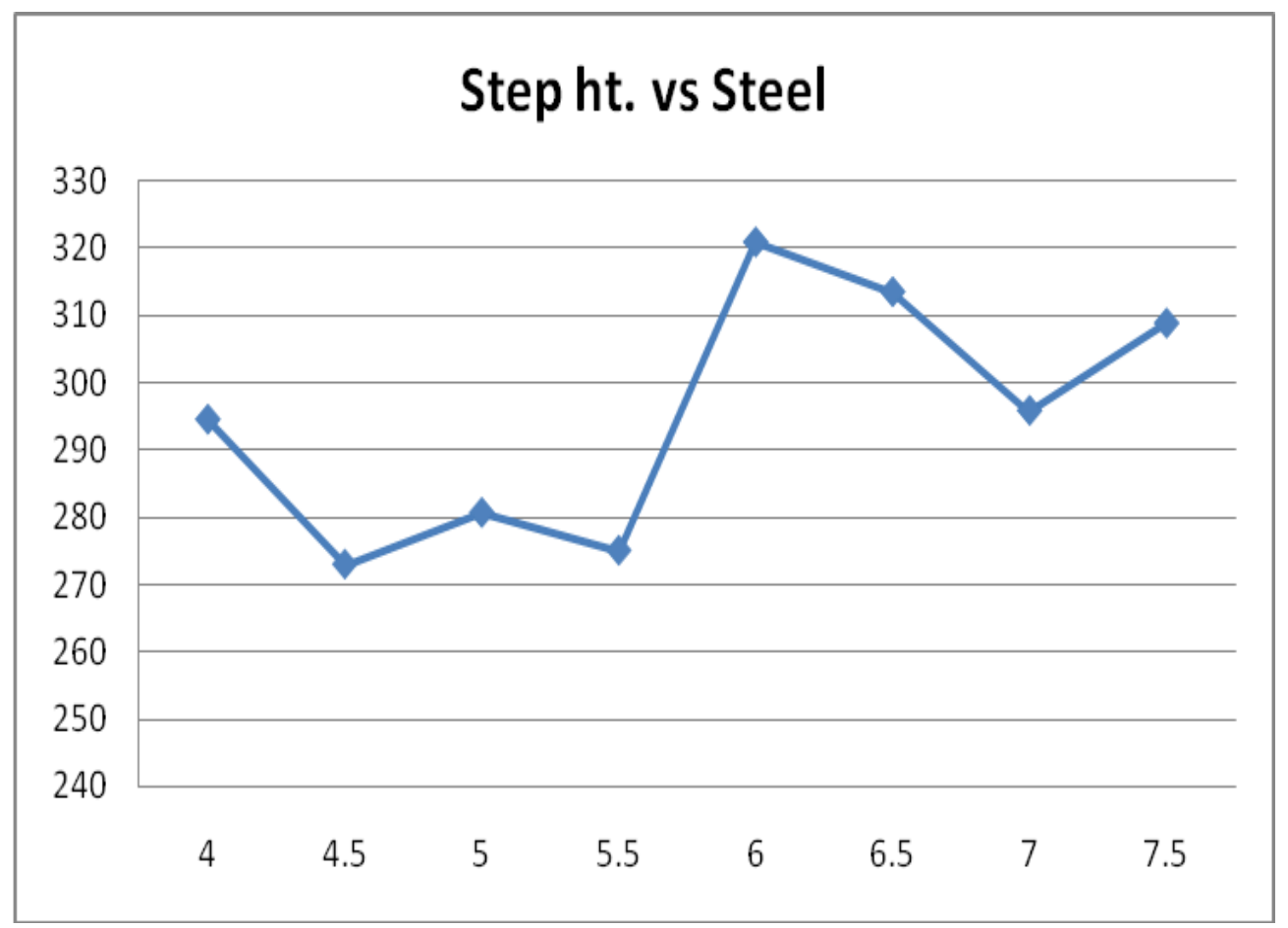

Graph 4: Step location Vs steel kg/m for wall Ht. 8m

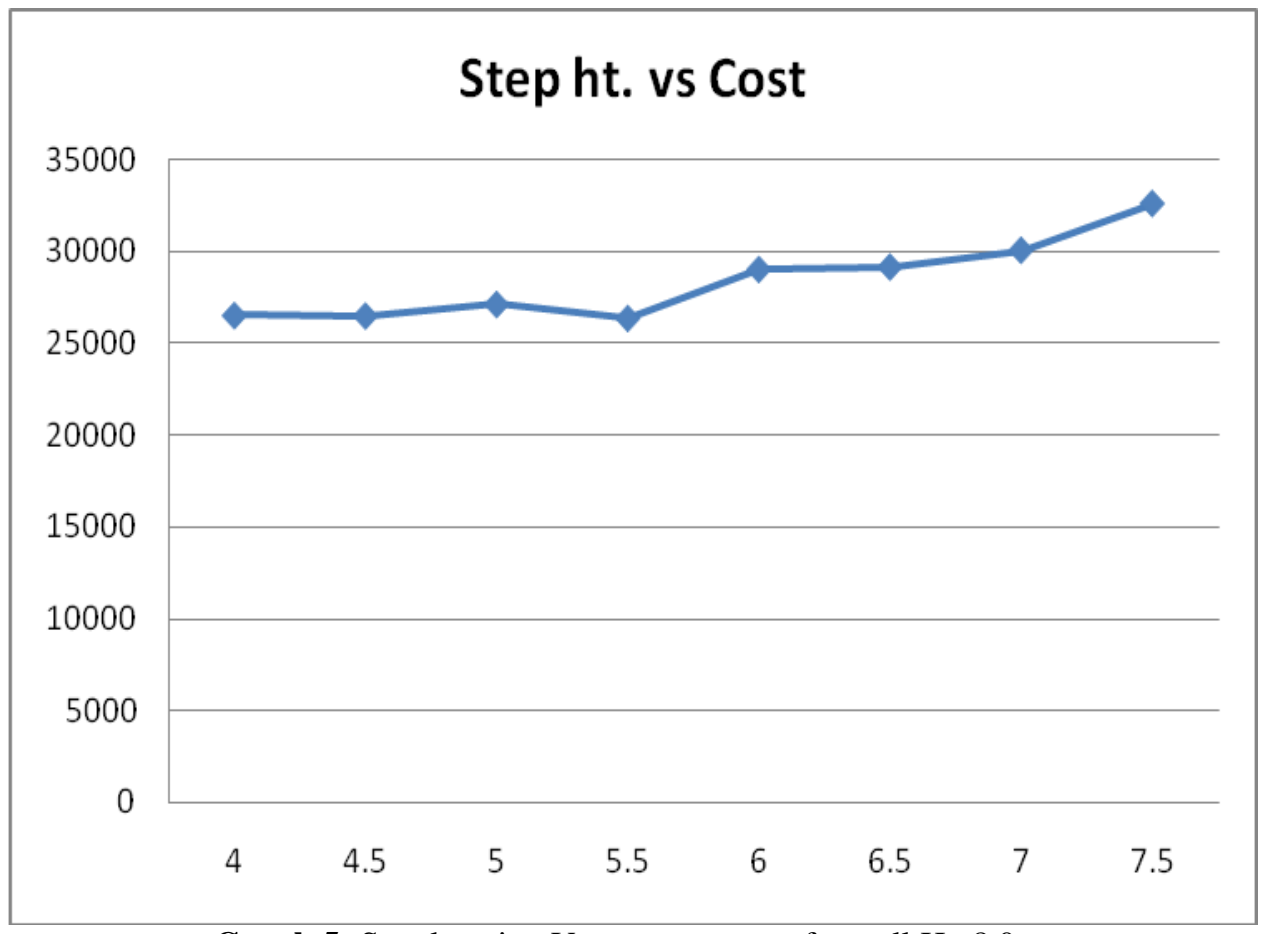

Graph 5: Step location Vs cost per meter for wall Ht. $8.0 \mathrm{~m}$

\section{3) Stepped Retaining Wall of Height 10m:}

\section{Assumptions}

1. Back fill is enough compacted.

2. Step length embedded in backfill $-5.5 \mathrm{~m}$

3. Step dimensions - $600 \times 300 \mathrm{~mm}$ 
Table 3: Stability analysis and cost comparison for wall hieght $10 \mathrm{~m}$.

\begin{tabular}{|l|l|l|l|l|}
\hline $\begin{array}{l}\text { Step from } \\
\text { top m. }\end{array}$ & $\begin{array}{l}\text { Width of toe } \\
\text { slab m }\end{array}$ & $\begin{array}{l}\text { Width of heel } \\
\text { slab m }\end{array}$ & $\begin{array}{l}\text { Depth of base } \\
\text { slab m }\end{array}$ & $\begin{array}{l}\text { Total base } \\
\text { slab m }\end{array}$ \\
\hline & & & & \\
\hline 5 & 1.5 & 8.5 & 0.55 & 10.5 \\
\hline 5.5 & 1.45 & 7.15 & 0.55 & 9.15 \\
\hline 6 & 1.4 & 6.85 & 0.55 & 8.75 \\
\hline 6.5 & 1.35 & 6.65 & 0.55 & 8.5 \\
\hline $\mathbf{7}$ & $\mathbf{1 . 5}$ & $\mathbf{6 . 5}$ & $\mathbf{0 . 5}$ & $\mathbf{8 . 5}$ \\
\hline 7.5 & 1.45 & 6.15 & 0.55 & 8.2 \\
\hline 8 & 1.5 & 5.55 & 0.55 & 7.8 \\
\hline 8.5 & 1.5 & 5.25 & 0.55 & 7.5 \\
\hline 9 & 1.6 & 4.925 & 0.6 & 7.25 \\
\hline 9.5 & 1.75 & 5.425 & 0.6 & 8 \\
\hline
\end{tabular}

Step ht. vs Concrete

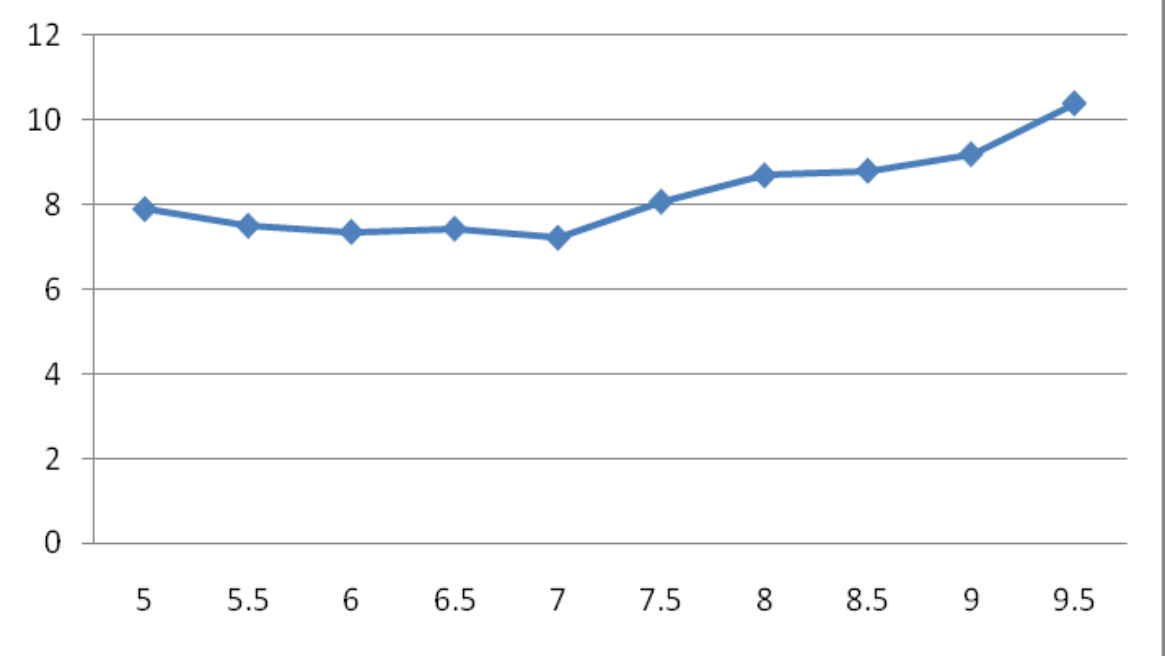

Graph 6: Step location Vs concrete cum/m for wall Ht. $8.0 \mathrm{~m}$.

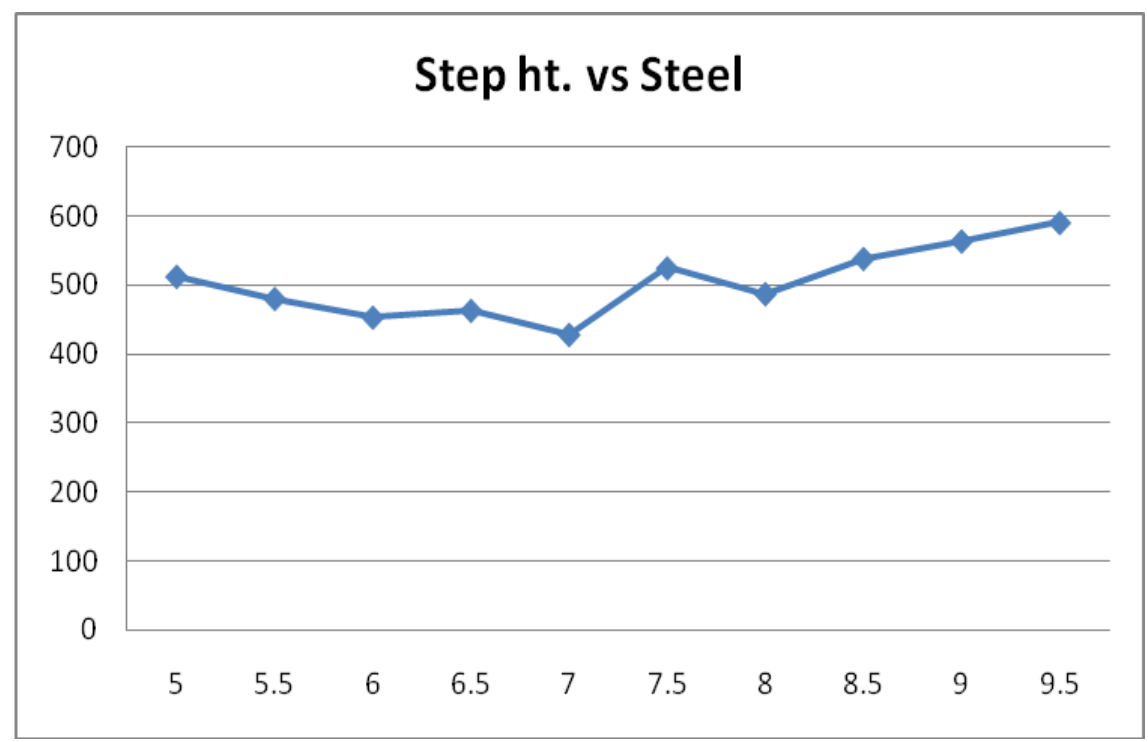

Graph 7: Step location Vs steel kg/m for wall Ht. 10.0 m 


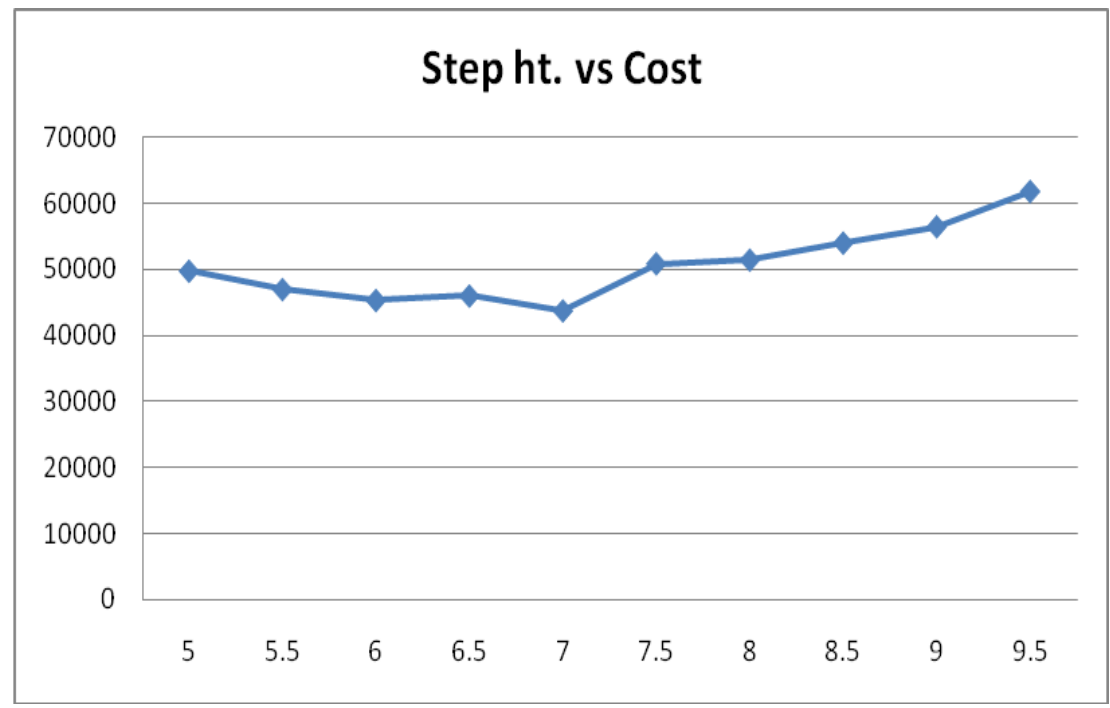

Graph 8: Step location Vs cost per meter for wall Ht. $10.0 \mathrm{~m}$

\section{4) Stepped Retaining Wall of Height 12m:}

\section{Assumptions}

1. Step length embedded in backfill - $6.5 \mathrm{M}$

2. Step dimensions - $650 \times 400 \mathrm{~mm}$

Table 4: Stability analysis and cost comparison for wall ht. $12 \mathrm{~m}$

\begin{tabular}{|l|l|l|l|l|}
\hline \multirow{2}{*}{$\begin{array}{l}\text { Step from } \\
\text { top }\end{array}$} & $\begin{array}{l}\text { Width of toe } \\
\text { slab m }\end{array}$ & $\begin{array}{l}\text { Width of heel } \\
\text { slab m }\end{array}$ & $\begin{array}{l}\text { Depth of base } \\
\text { slab m }\end{array}$ & $\begin{array}{l}\text { Total base } \\
\text { slab m }\end{array}$ \\
\cline { 1 - 2 } \cline { 5 - 5 } & 2.1 & 5.9 & 0.75 & 8.65 \\
\hline 6.5 & 1.9 & 6.6 & 0.75 & 9.15 \\
\hline 7 & 1.8 & 6.3 & 0.65 & 8.75 \\
\hline 7.5 & 1.65 & 6.2 & 0.65 & 8.45 \\
\hline 8 & 1.5 & 6.35 & 0.65 & 8.45 \\
\hline 8.5 & 1.4 & 6.1 & 0.65 & 8.2 \\
\hline 9 & 1.25 & 5.85 & 0.6 & 7.8 \\
\hline 9.5 & 1.4 & 5.45 & 0.7 & 7.5 \\
\hline 10 & 1.51 & 5.09 & 0.7 & 7.25 \\
\hline 10.5 & 1.6 & 5.6 & 0.65 & 8 \\
\hline 11 & 1.7 & 5.2 & 0.65 & 7.8 \\
\hline 11.5 & 1.8 & 5.225 & 0.7 & 8.1 \\
\hline
\end{tabular}

\begin{tabular}{|l|l|l|l|l|l|}
\hline $\begin{array}{l}\text { Upward soil } \\
\text { pressure } \\
\text { KN/m2 }\end{array}$ & $\begin{array}{l}\text { Effective } \\
\text { frictional } \\
\text { force KN }\end{array}$ & Concretem3 & $\begin{array}{l}\text { Steel } \\
\text { quantity } \\
\mathrm{Kg} / \mathrm{m}\end{array}$ & Cost Rs/m \\
\hline Pmax. & Pmin. & & & & \\
\hline 297.29 & 217.93 & 213.27 & 9.4875 & 780.71 & 66776.78 \\
\hline 295.99 & 229.29 & 252.98 & 10.1125 & 653.98 & 63514.89 \\
\hline 297.01 & 224.59 & 296.07 & 9.1875 & 664.01 & 60708.68 \\
\hline 299.91 & 240.15 & 342.53 & 9.055 & 602.19 & 57586.67 \\
\hline 295.05 & 249.66 & 392.37 & 9.2925 & 665.24 & 61129.07 \\
\hline
\end{tabular}




\begin{tabular}{|l|l|l|l|l|l|}
286.61 & 260.06 & 445.59 & 9.7925 & 704.89 & 64584.02 \\
\hline 270.77 & 280.76 & 502.18 & 9.405 & 855.36 & 69697.98 \\
\hline 255.28 & 289.2 & 562.14 & 10 & 856.7 & 71838.1 \\
\hline 240.75 & 294.08 & 562.14 & 10.075 & 861.16 & 72292.38 \\
\hline 245.75 & 283.49 & 692.2 & 11.2375 & 810.15 & 74167.7 \\
\hline 244.36 & 283.43 & 762.29 & 11.945 & 822.89 & 77191.77 \\
\hline 249.26 & 283.24 & 835.76 & 11.85125 & 877.55 & 79214.025 \\
\hline
\end{tabular}

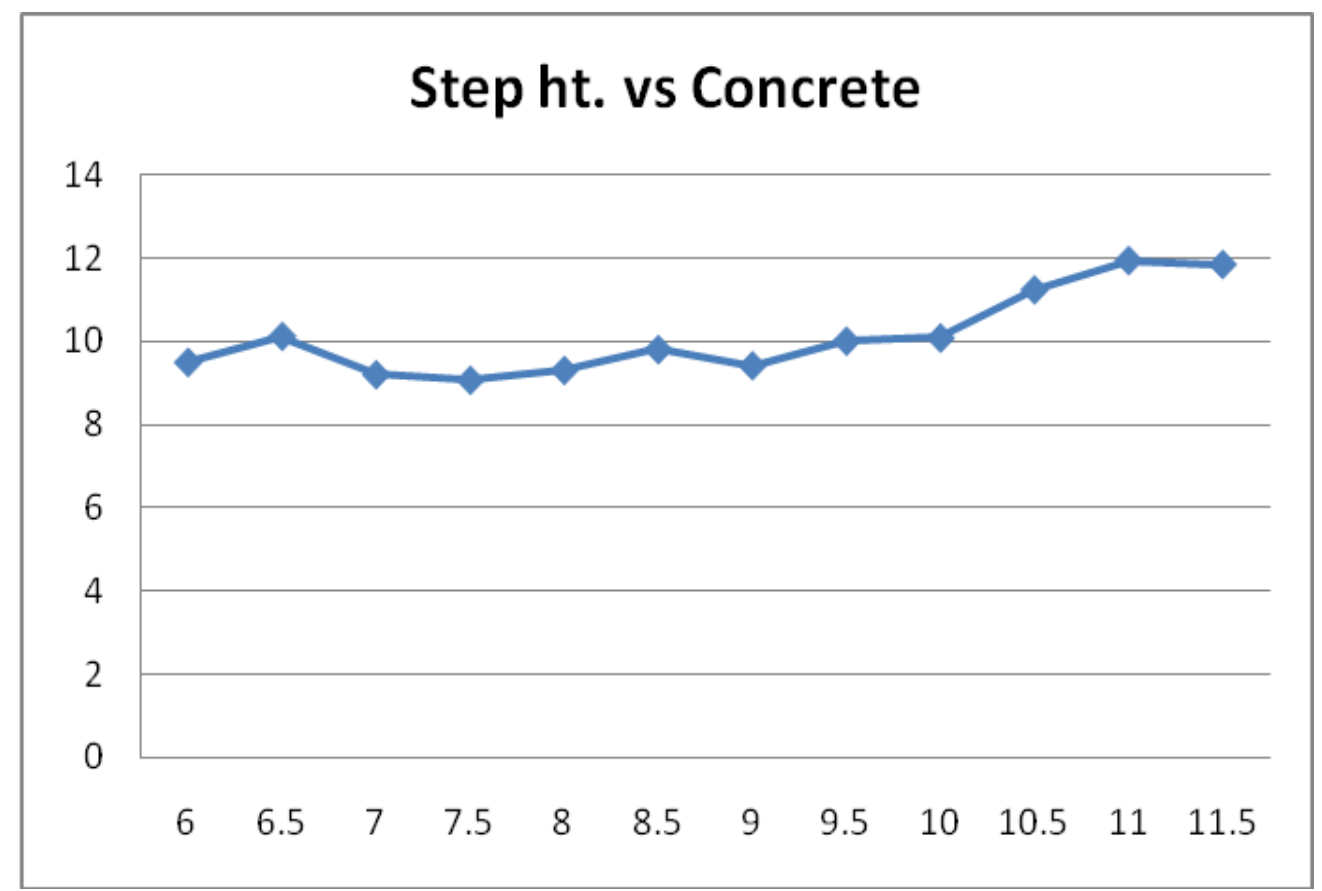

Graph 9: Step location Vs concrete cum/m for wall Ht. 12m

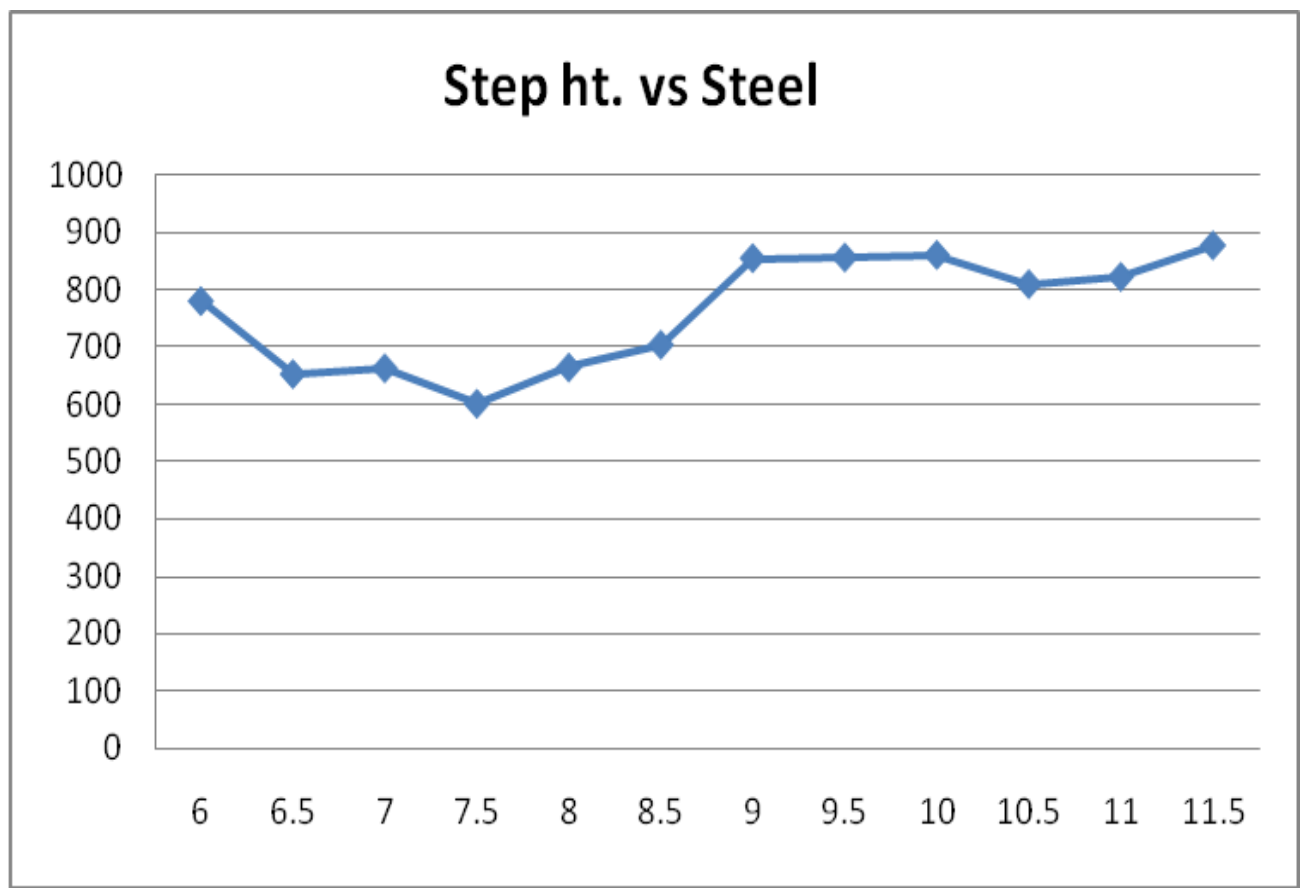

Graph 10: Step location Vs steel kg/m for wall Ht. $12.0 \mathrm{~m}$ 


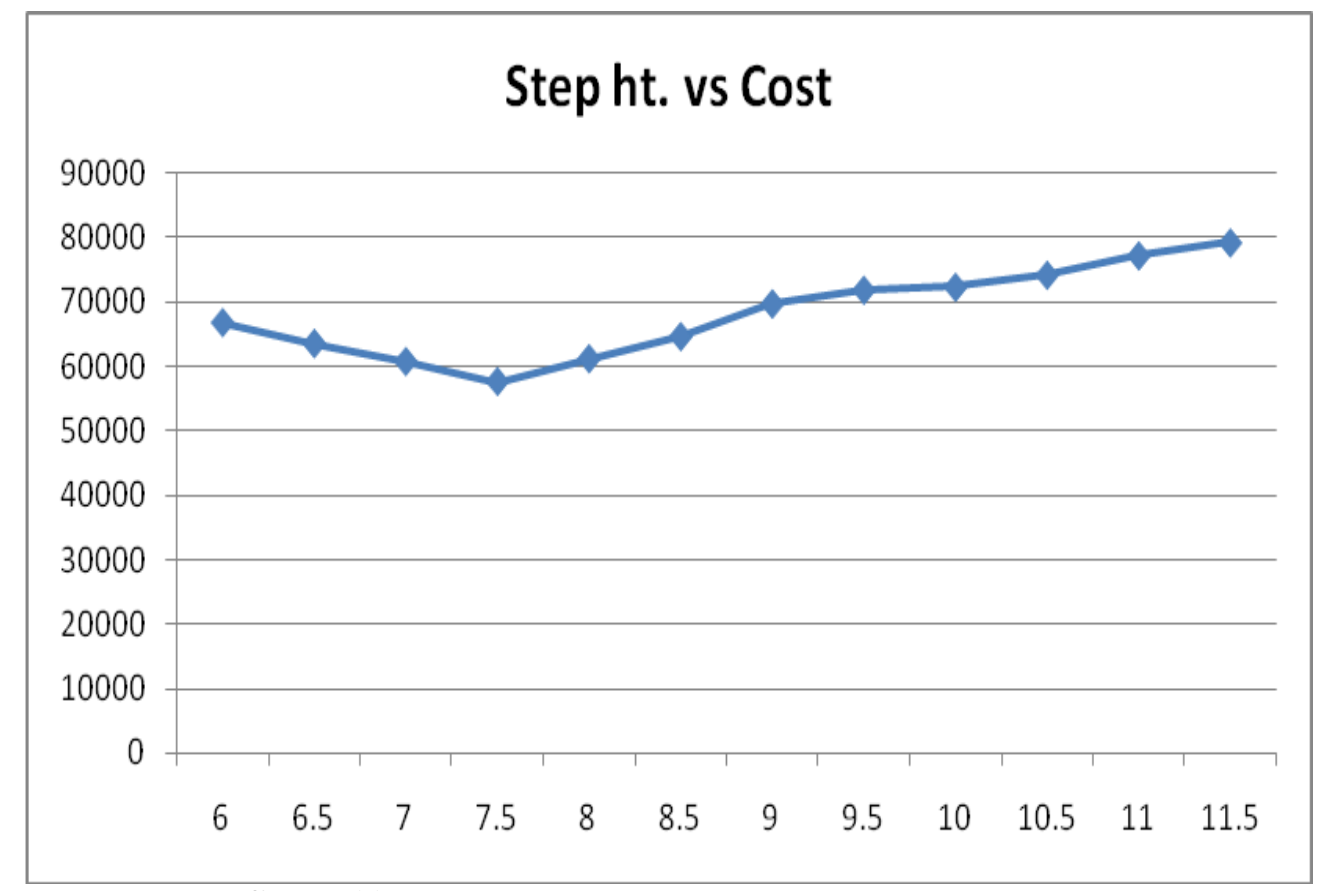

Graph 11: Step location Vs cost per meter for wall Ht. 8.0 m

\section{5) Stepped Retaining Wall of Height 15m}

\section{Assumptions}

1. Step length embedded in backfill $-7.5 \mathrm{~m}$

2. Step dimensions - $700 \times 450 \mathrm{~mm}$

Table 5: Stability analysis and cost comparison for wall ht.15m

\begin{tabular}{|c|c|c|c|c|c|c|}
\hline \multirow{2}{*}{$\begin{array}{c}\text { Step } \\
\text { from } \\
\text { top m }\end{array}$} & $\begin{array}{c}\text { Width } \\
\text { of toe } \\
\text { slab } \mathrm{m}\end{array}$ & $\begin{array}{c}\text { Width } \\
\text { of } \\
\text { heel } \\
\text { slab } \\
\mathrm{m}\end{array}$ & $\begin{array}{c}\text { Depth } \\
\text { of } \\
\text { base } \\
\text { slab } \\
\mathrm{m}\end{array}$ & $\begin{array}{c}\text { Total } \\
\text { base slab } \\
\mathrm{m}\end{array}$ & $\begin{array}{c}\text { Stem } \\
\text { thickness } \\
\text { in } \mathrm{m} \\
\text { top }\end{array}$ & Bottom. \\
\cline { 1 - 1 } & 2.1 & 6.3 & 0.75 & 9.5 & 0.35 & 1.1 \\
\hline 8 & 2.15 & 6.1 & 0.75 & 9.15 & 0.35 & 0.9 \\
\hline 8.5 & 2.12 & 5.73 & 0.75 & 8.75 & 0.35 & 0.9 \\
\hline 9 & 2.12 & 5.46 & 0.75 & 8.5 & 0.35 & 0.92 \\
\hline 9.5 & 2 & 5.33 & 0.72 & 8.15 & 0.35 & 0.82 \\
\hline 10 & 2 & 5.225 & 0.8 & 8.195 & 0.35 & 0.97 \\
\hline 10.5 & 2 & 4.7 & 0.75 & 7.8 & 0.35 & 1.1 \\
\hline 11 & 2.1 & 4.15 & 0.75 & 7.5 & 0.35 & 1.25 \\
\hline 11.5 & 2.2 & 3.75 & 0.75 & 7.25 & 0.35 & 1.3 \\
\hline 12 & 2.25 & 4.45 & 0.75 & 8 & 0.35 & 1.3 \\
\hline 12.5 & 2.4 & 4.1 & 0.75 & 7.8 & 0.35 & 1.3 \\
\hline 13 & 2.6 & 4.2 & 0.785 & 8.1 & 0.35 & 1.3 \\
\hline 13.5 & 2.8 & 4.4 & 0.85 & 8.5 & 0.35 & 1.3 \\
\hline 14 & 2.9 & 5.1 & 0.9 & 9.3 & 0.35 & 1.3 \\
\hline 14.5 & 3 & 6.05 & 1 & 10.4 & 0.35 & 1.35 \\
\hline
\end{tabular}




\begin{tabular}{|l|l|l|l|l|l|}
\hline $\begin{array}{l}\text { Upward } \\
\text { soil } \\
\text { pressure } \\
\text { KN/m2 }\end{array}$ & $\begin{array}{l}\text { Effective } \\
\text { frictional } \\
\text { force KN }\end{array}$ & Concretem3 & $\begin{array}{l}\text { Steel } \\
\text { quantity } \\
\text { Kg/m }\end{array}$ & Cost Rs/m \\
\hline Pmax. & Pmin. & & & & \\
\hline 297.9 & 290.8 & 299.8 & 12.5625 & 928.64 & 83900.27 \\
\hline 298.52 & 284.82 & 348.86 & 11.8625 & 968.68 & 83171.99 \\
\hline 295.9 & 282.2 & 401 & 11.875 & 946 & 82240.5 \\
\hline 291.9 & 275 & 457.9 & 12.09 & 925.5 & 82111.5 \\
\hline 300 & 283.5 & 517.88 & 11.4255 & 967 & 81570.25 \\
\hline 292.9 & 272.8 & 581.5 & 13.156 & 1015.9 & 89729.7 \\
\hline 282 & 280.35 & 648.73 & 13.4625 & 1004 & 90290.75 \\
\hline 264.5 & 276.8 & 719.6 & 14.425 & 1111 & 98260.5 \\
\hline 238.88 & 283.5 & 794.12 & 14.925 & 1089 & 99064.5 \\
\hline 267.8 & 271.9 & 872.19 & 15.9 & 1233 & 108669 \\
\hline 234.5 & 286.35 & 954 & 16.1625 & 1167 & 106749.75 \\
\hline 223.5 & 290.75 & 1040 & 17.0835 & 1064 & 105544.25 \\
\hline 213 & 299 & 1128.5 & 18.3625 & 1077 & 110579.75 \\
\hline 225 & 300 & 1221 & 19.92 & 1350 & 127770 \\
\hline 246.25 & 300 & 1317.5 & 22.725 & 1511 & 144510.5 \\
\hline
\end{tabular}

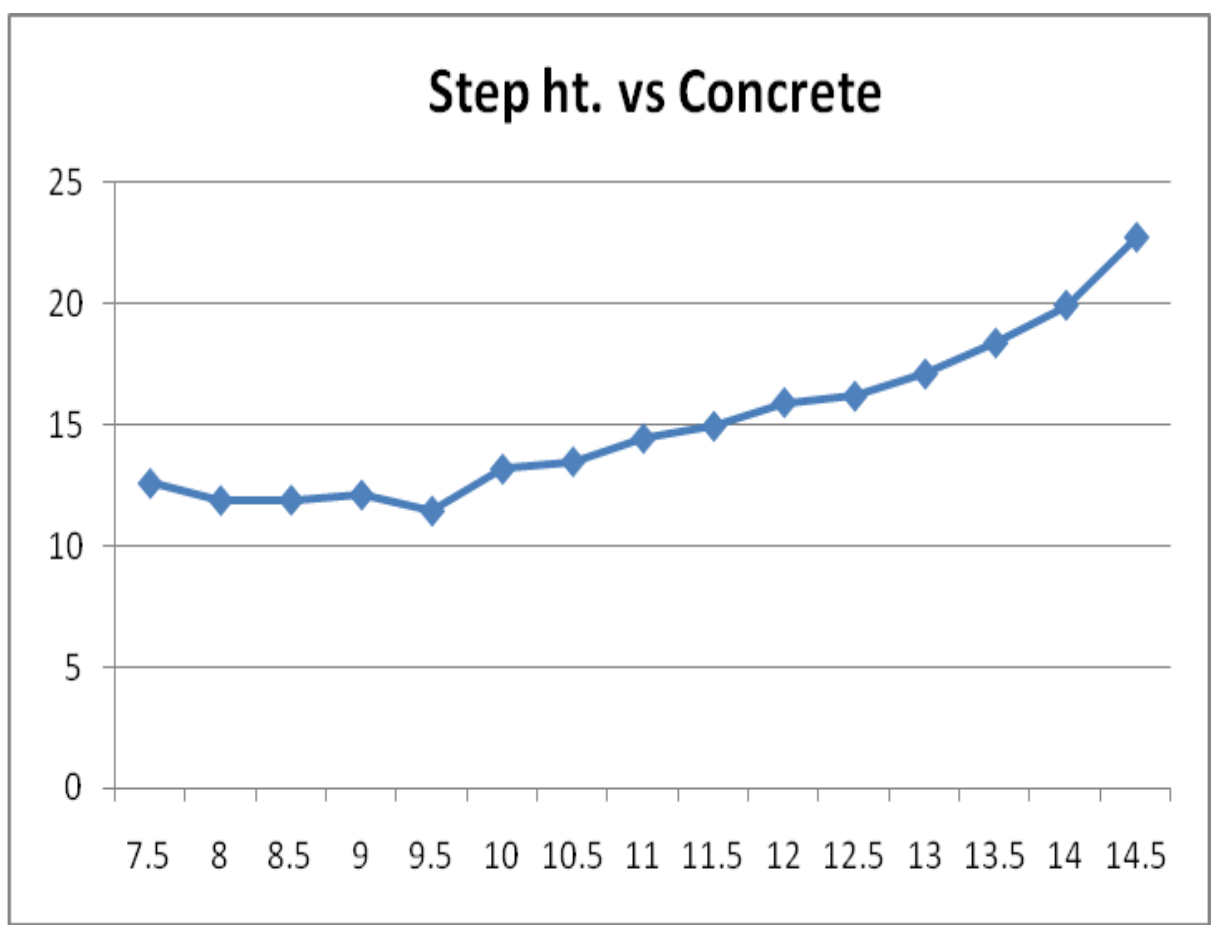

Graph 12: Step location Vs concrete cum/m for wall Ht. $15.0 \mathrm{~m}$ 


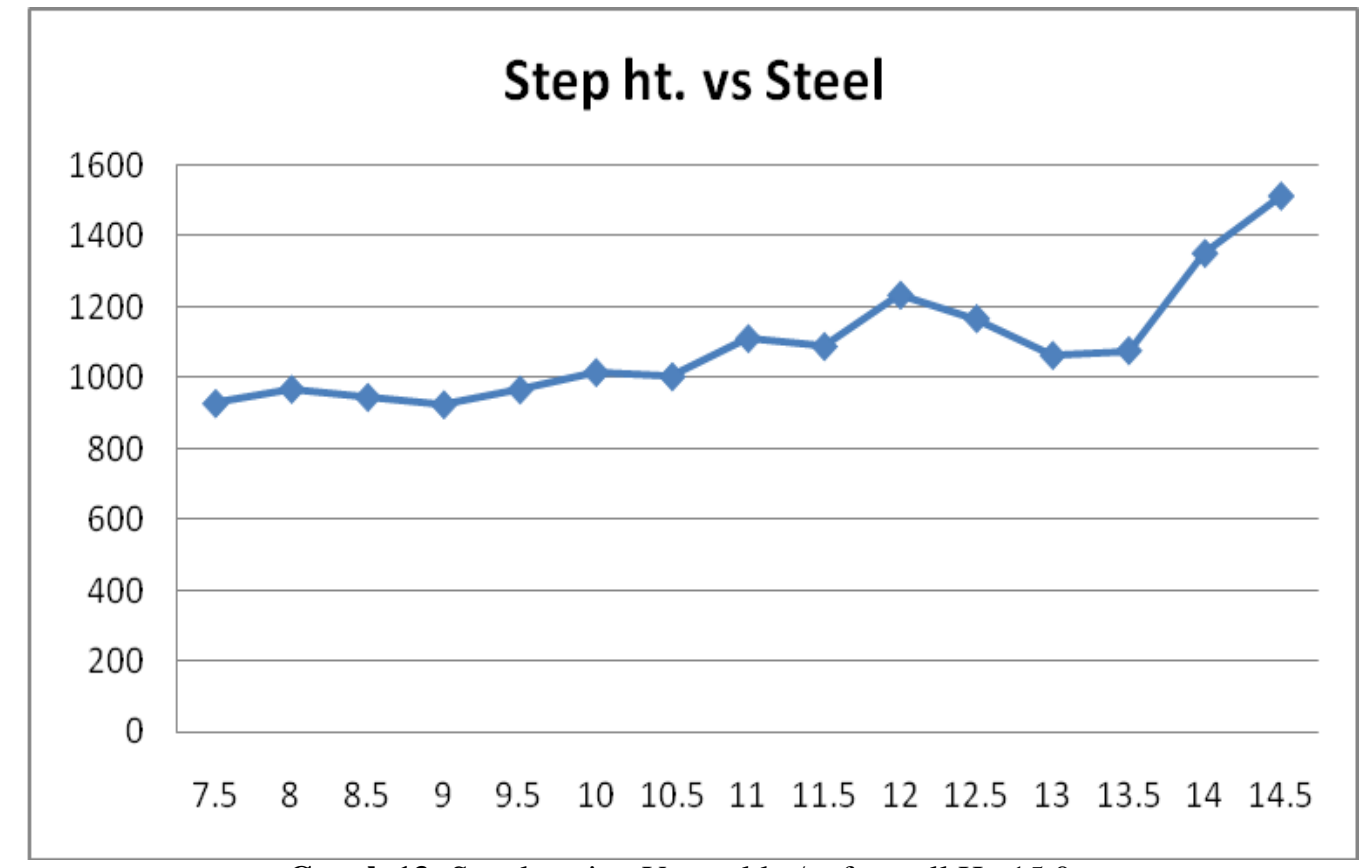

Graph 13: Step location Vs steel $\mathrm{kg} / \mathrm{m}$ for wall Ht. $15.0 \mathrm{~m}$

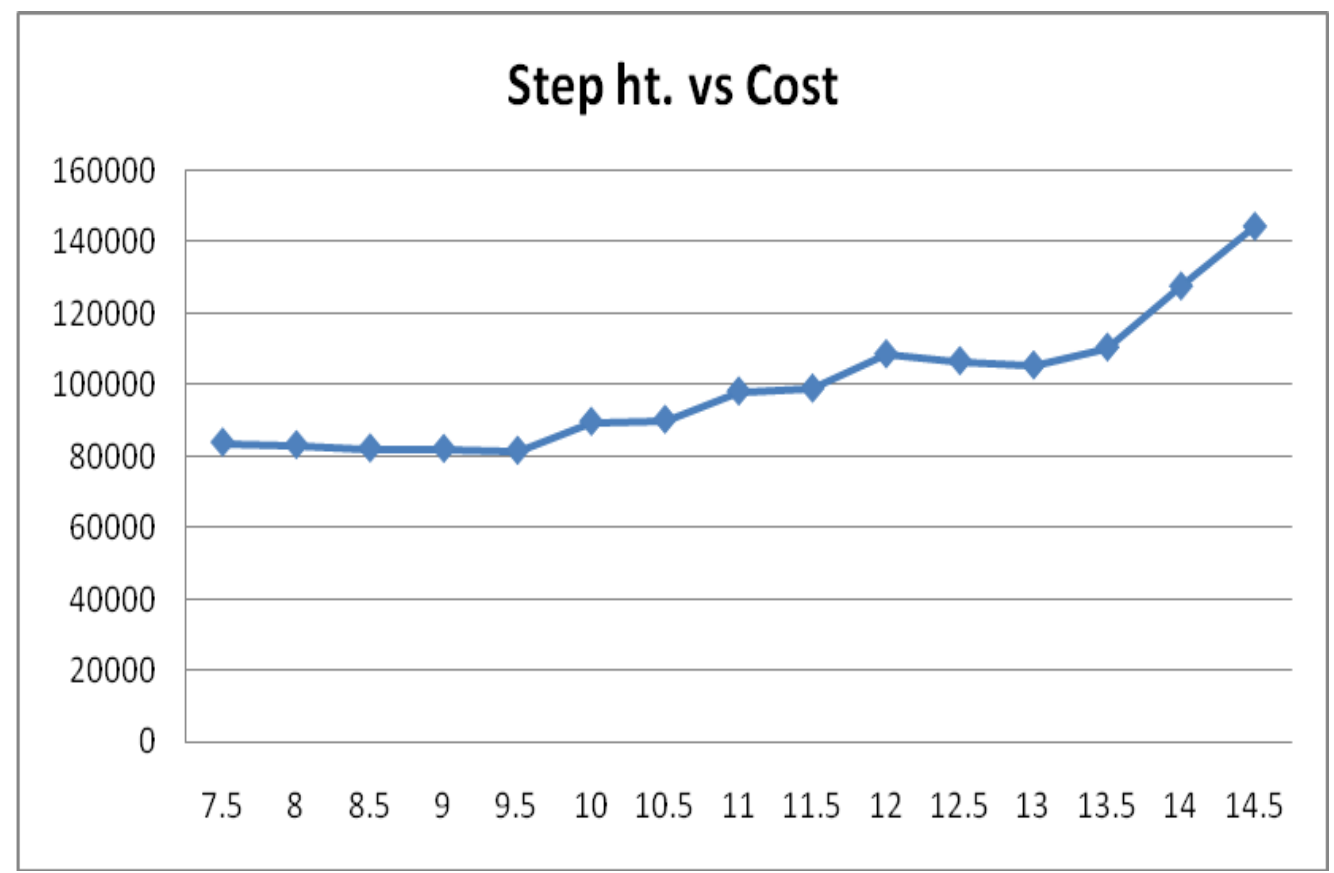

Graph 14: Step location Vs cost per meter for wall Ht. 15.0m

\section{RESULTS AND DISSCUSSIONS}

\section{Example}

The example of analysis and design of stepped cantilever retaining wall are given below.

\section{Data Assumptions}

Data assumed for the stability calculation of stepped cantilever retaining wall

- Free Board not necessary

- The backfill is enough compacted to develop necessary friction.

- Bearing Capacity of soil: $300 \mathrm{KN} / \mathrm{Sq}$. m

- Water level is much below the level of base and effect of soil moisture is ignored.

- Dry density of soil: $18 \mathrm{KN} / \mathrm{Cubic} \mathrm{m}$.

- Angle of internal friction: 300

- Coefficient of friction: 0.60 
- Stability is checked for sliding and overturning.

- Factor of safety against sliding $=1.5$

- Factor of safety against overturning $=2.0$

The moment and reinforcement provided for various heights are as shown in table

\subsection{Counter fort Retaining wall}

The structural analysis of counter fort Retaining wall is done as per routine analytical practices. Generally these walls are use for span more than $6 \mathrm{~m}$, but here in order to compare the results analysis and design of these counter fort retaining walls is done for Heights $6 \mathrm{~m}$ to $15 \mathrm{~m}$. The mechanism of this wall is different from cantilever wall and here Base slab is more important design aspect.

Table 6: Dimensions of Counter fort Retaining Wall

\begin{tabular}{|c|c|c|c|c|c|c|}
\hline \multirow{2}{*}{$\begin{array}{l}\text { Ht of } \\
\text { wall } \\
\text { m }\end{array}$} & \multirow{2}{*}{$\begin{array}{l}\text { Total } \\
\text { Base Slab } \\
\text { M }\end{array}$} & \multirow{2}{*}{$\begin{array}{l}\text { Width } \\
\text { of Toe } \\
\text { Slab }\end{array}$} & \multirow{2}{*}{$\begin{array}{l}\text { Width } \\
\text { of Heel } \\
\text { slab }\end{array}$} & \multirow{2}{*}{$\begin{array}{l}\text { Base } \\
\text { slab } \\
\text { Thk. M }\end{array}$} & \multicolumn{2}{|c|}{ Stem Thk. m } \\
\hline & & & & & Top & Botm. \\
\hline 6 & 3.5 & 0.3 & 3.0 & 0.28 & 0.2 & 0.2 \\
\hline 8 & 4.25 & 0.5 & 3.45 & 0.35 & 0.3 & 0.3 \\
\hline 10 & 5.6 & 1.0 & 4.25 & 0.45 & 0.45 & 0.35 \\
\hline 12 & 7.75 & 1.25 & 6.05 & 0.5 & 0.45 & 0.45 \\
\hline 15 & 10.0 & 2.75 & 6.70 & 0.77 & 0.55 & 0.55 \\
\hline
\end{tabular}

Counter fort Details

\begin{tabular}{|l|l|l|l|l|l|}
\hline Spacing & 4.0 & 3.5 & 3.0 & 3.0 & 3.0 \\
\hline Thickness & 0.3 & 0.375 & 0.4 & 0.45 & 0.55 \\
\hline
\end{tabular}

The analysis of Base slab for wall is presented in table Here Toe slab is designed as cantilever slab spanning from stem. The upward soil pressure will be act as major load on toe slab. But the heel slab will be designed as simply supported slab in between two adjacent counter forts. Sometimes when toe projection is larger and if there is possibility of stress reversal in stem, the counter forts are also provided on toe slab at that time Toe slab design will also be as heel slab design .The major load for heel slab will be effective load from average Upward pressure and Retained soil load on heel slab.

The base slab depth is provided as per required for maximum Bending Moment while reinforcement is provided as per actual requirement for Toe and Heel slab.

Table 7: Structural Analysis of Counter-fort Retaining wall (Base slab)

\begin{tabular}{|l|l|l|l|l|}
\hline \multirow{2}{*}{$\begin{array}{l}\text { Height of } \\
\text { wall } \\
\mathrm{m}\end{array}$} & \multicolumn{2}{|l|}{$\begin{array}{l}\text { Bending moment } \\
\text { (KN.m) }\end{array}$} & $\begin{array}{l}\text { Depth of } \\
\text { base slab } \\
\text { required } \mathrm{mm}\end{array}$ & $\begin{array}{l}\text { Depth of } \\
\text { base slab } \\
\text { Provided } \mathrm{mm}\end{array}$ \\
\cline { 2 - 5 } & Toe & Heel & 240.03 & 400 \\
\hline 6 & 12.67 & 158.98 & 290.00 & 450 \\
\hline 8 & 47.58 & 232.12 & 390.00 & 550 \\
\hline 10 & 187.55 & 419.80 & 440.00 & 600 \\
\hline 12 & 288.36 & 534.34 & 710.00 & 850 \\
\hline 15 & 1152.18 & 1391.32 & & \\
\hline
\end{tabular}

The reinforcement provided for base slab i.e. Toe slab and various locations is shown in table 8

Table 8: Design of Base slab of counter fort retaining wall

\begin{tabular}{|l|l|l|l|l|l|l|l|}
\hline \multirow{3}{*}{$\begin{array}{l}\text { Ht. Of } \\
\text { wall } \\
\text { m. }\end{array}$} & $\begin{array}{l}\text { Base slab } \\
\text { Thick. }\end{array}$ & Mm & Main Steel. & Toe slab & Heel slab & \\
\cline { 3 - 8 } & Ast. $\quad \mathrm{mm} 2$ & $\begin{array}{l}\text { Bar Dia. } \\
\text { Spacing }\end{array}$ & Ast. mm2 & $\begin{array}{l}\text { Bar Dia. } \\
\text { Spacing }\end{array}$ \\
\hline
\end{tabular}




\begin{tabular}{|l|l|l|l|l|l|}
\hline 6 & 400 & 168.73 & $\begin{array}{l}\phi 10 \\
@ 150 \mathrm{~mm}\end{array}$ & 1172.70 & $\begin{array}{l}\phi 20 \\
@ 150 \mathrm{~mm}\end{array}$ \\
\hline 8 & 450 & 297.07 & $\begin{array}{l}\Phi 12 \\
@ 150 \mathrm{~mm}\end{array}$ & 1538.54 & $\phi 20 \quad @ 150 \mathrm{~mm}$ \\
\hline 10 & 550 & 981.27 & $\begin{array}{l}\Phi 16 \\
@ 150 \mathrm{~mm}\end{array}$ & 2317.76 & $\Phi 25 \quad @ 150 \mathrm{~mm}$ \\
\hline 12 & 600 & 1399.52 & $\begin{array}{l}\Phi 20 \\
@ 150 \mathrm{~mm}\end{array}$ & 2724.55 & $\begin{array}{l}\Phi 25 \\
@ 150 \mathrm{~mm}\end{array}$ \\
\hline 15 & 850 & 4183.46 & $\begin{array}{l}\Phi 25 \\
@ 115 \mathrm{~mm}\end{array}$ & 5194.55 & $\begin{array}{l}\Phi 32 \\
@ 150 \mathrm{~mm}\end{array}$ \\
\hline
\end{tabular}

The mechanism of stem of counter fort retaining wall and Cantilever retaining wall is not same. In cantilever retaining wall, stem was acting as free cantilever with span equal height of wall while in counter fort, stem acts as simply supported slab spanning in between two adjacent counter forts. The effective span for this will be span of counter fort along length of wall. The dimensions of stem are reduced due to this mechanism. The bending moment of the vertical wall is maximum at the junction of stem (wall) with Base and reduces to the zero at the top of the wall.

The moments and reinforcement provided for various heights are as shown in table 9

Table 9: Moment and Reinforcement details along length of stem for counter fort wall

\begin{tabular}{|c|c|c|c|c|c|c|}
\hline \multirow{3}{*}{$\begin{array}{l}\text { Ht. of } \\
\text { wall } \\
\text { m. }\end{array}$} & \multirow{3}{*}{$\begin{array}{l}\text { Moments } \\
\text { length } \\
\text { stem }(\mathrm{KNm})\end{array}$} & \multicolumn{5}{|c|}{ Steel prov. In Vertical wall } \\
\hline & & \multicolumn{2}{|c|}{ Stem Thickness } & & & \\
\hline & & Dreq. mm & $\begin{array}{l}\text { Dprov. } \\
\mathrm{mm}\end{array}$ & Ast $\mathrm{mm} 2$ & \multicolumn{2}{|c|}{ Bar Dia. \& Spacing } \\
\hline 6 & 72 & 161.51 & 200 & 1130.09 & $\Phi 10$ & @70mm \\
\hline 8 & 73.5 & 163.19 & 300 & 1736.00 & $\Phi 12$ & $65 \mathrm{~mm}$ \\
\hline 10 & 67.5 & 156.39 & 350 & 552.52 & $\Phi 16$ & @150mm \\
\hline 12 & 81 & 171.31 & 450 & 510.83 & $\Phi 20$ & @ 150mm \\
\hline 15 & 101.25 & 191.53 & 550 & 520.35 & $\Phi 25$ & @ 150mm \\
\hline
\end{tabular}

The counter forts act as self-supporting structural elements for retaining wall. It takes reactions, both from the stem as well as Heel slab. The counter fort may be considered to bend as a cantilever, fixed at heel slab. The counter fort acts as an inverted $T$ beam of varying rib depth. The structural analysis of counter fort is done based on above assumptions. The Max. Depth of this cantilever beam is width of heel slab. The steel reinforcement is provided as per requirement for tensile stress induced in it due to soil load on stem.

The moments and connection of counter fort details for various wall heights are as shown in table 10

Table 10: Moment and Connections of counter fort with Heel slab

\begin{tabular}{|l|l|lc|l|l|l|}
\hline \multirow{2}{*}{ Stamm. } & \multirow{2}{*}{ Moment } & \multirow{2}{*}{ Bar Dia. And Spacing } & \multicolumn{3}{|l}{ Connections of counter fort with Heel Slab } \\
\cline { 5 - 8 } & & & & Horizontal Force & $\begin{array}{l}\text { Bar } \\
\text { Dia. }\end{array}$ & $\begin{array}{l}\text { Spacing of } \\
\text { Stirrups }\end{array}$ \\
\hline 6 & 864 & $\Phi 20$ & $@ 100 \mathrm{~mm}$ & 144 & $\Phi 8$ & $100 \mathrm{~mm}$ \\
\hline 8 & 1792 & $\Phi 20$ & $@ 100 \mathrm{~mm}$ & 168 & $\Phi 8$ & $100 \mathrm{~mm}$ \\
\hline 10 & 3000 & $\Phi 25$ & $@ 100 \mathrm{~mm}$ & 180 & $\Phi 10$ & $100 \mathrm{~mm}$ \\
\hline 12 & 5184 & $\Phi 25$ & $@ 100 \mathrm{~mm}$ & 216 & $\Phi 10$ & $100 \mathrm{~mm}$ \\
\hline 15 & 10125 & $\Phi 32$ & $@ 100 \mathrm{~mm}$ & 270 & $\Phi 12$ & $100 \mathrm{~mm}$ \\
\hline
\end{tabular}


The main stress along counter fort is tensile. The connection of counter fort with base slab and stem is important for all assumed mechanism. The steel reinforcement provided is in the form of two legged stirrups of required diameter steel. The saving in steel reinforcement can be done as per curtailment / Reduction in number of stirrups from bottom to top side of wall.

\subsection{Stepped Cantilever Retaining Wall}

The stepped cantilever wall is new type suggested in this thesis. Here concrete steps are provided on stem projecting into backfill. The pressure compacted backfill will anchor the concrete plate/step and will develop frictional resistance force; this will act as indirect support for cantilever retaining wall. In short stem will act as propped cantilever and thus will reduce the destructive forces on stem / retaining wall.

Table 11: Summary of Dimensions of Stepped Cantilever Retaining Wall

\begin{tabular}{|l|l|l|l|l|l|l|}
\hline \multirow{2}{*}{ Ht. of wall $\mathrm{m}$} & $\begin{array}{l}\text { Total } \\
\text { base } \\
\text { slab } \\
\mathrm{m}\end{array}$ & $\begin{array}{l}\text { Width of Toe } \\
\text { Slab } \\
\mathrm{M}\end{array}$ & $\begin{array}{l}\text { Width of } \\
\text { Heel slab } \mathrm{m}\end{array}$ & $\begin{array}{l}\text { Rase slab } \\
\text { Thk. } \\
\mathrm{m}\end{array}$ & \multicolumn{2}{|l|}{ Stem Thickness } \\
\cline { 3 - 7 } & 2.85 & 0.65 & 1.9 & 0.4 & 0.2 & 0.3 \\
\hline 6 & 5.25 & 0.95 & 3.9 & 0.4 & 0.2 & 0.4 \\
\hline 8 & 6.5 & 1.5 & 4.4 & 0.60 & 0.25 & 0.6 \\
\hline 10 & 8.5 & 1.65 & 6.2 & 0.65 & 0.3 & 0.65 \\
\hline 12 & 10.5 & 2.0 & 7.3 & 0.9 & 0.5 & 1.2 \\
\hline 15 & & & & & & Bot \\
\hline
\end{tabular}

\begin{tabular}{|l|l|l|l|l|}
\hline Concrete Steps & Spacing & $\begin{array}{l}\text { From } \\
\text { Top }\end{array}$ & $\begin{array}{l}\text { Width } \\
\text { m }\end{array}$ & $\begin{array}{l}\text { Depth } \\
\text { m }\end{array}$ \\
\hline 3.5 & 2 & 4.00 & 0.45 & 0.3 \\
\hline 4.5 & 2 & 6.0 & 0.45 & 0.5 \\
\hline 6.0 & 2 & 8 & 0.6 & 0.5 \\
\hline 6.0 & 1.5 & 8 & 0.6 & 0.65 \\
\hline 5.75 & 1.25 & 7.75 & 0.75 & 0.7 \\
\hline
\end{tabular}

There is reduced soil load on base slab of wall firstly due to decreased base slab width and secondly due to reduction in load of soil resting on concrete steps/plates in backfill. In this case of wall interestingly it was the case that, wall was stable at shorter dimensions but the stem was pulled inside backfill due to assumed frictional force hence the structural dimensions were not much reduced to keep balance between self weight and resisting forces.

The forces acting and analysis and design of base slab for this new stepped cantilever retaining wall are as shown in Table12

Table 12: Structural Analysis of Stepped cantilever Retaining wall (Base slab)

\begin{tabular}{|l|l|l|l|l|}
\hline \multirow{2}{*}{$\begin{array}{l}\text { Ht. Of } \\
\text { wall } \mathrm{m} .\end{array}$} & $\begin{array}{l}\text { Bending moment } \\
(\mathrm{KNm})\end{array}$ & $\begin{array}{l}\text { Thickness } \\
\text { Required } \\
\text { mm }\end{array}$ & $\begin{array}{l}\text { Thickness } \\
\text { Provided } \\
\text { Mm }\end{array}$ \\
\cline { 2 - 5 } & Toe & Heel & 195.05 & 400 \\
\hline 6 & 72.03 & 105.00 & 538.68 & 650 \\
\hline 8 & 205.34 & 800.88 & 598.18 & 750 \\
\hline 10 & 581.84 & 987.57 & 618.55 & 800 \\
\hline 15 & 656.00 & 1112.92 & 750.15 & 900 \\
\hline
\end{tabular}

Table 13: Design of Base slab of Stepped cantilever Retaining wall

\begin{tabular}{|l|l|l|l|l|l|}
\hline \multirow{2}{*}{$\begin{array}{l}\text { Ht. of } \\
\text { wall } \\
\mathrm{m} .\end{array}$} & \multirow{2}{*}{$\begin{array}{l}\text { Base slab } \\
\text { Thick. }\end{array}$} & Mm & \multicolumn{4}{|l|}{ Main Steel. } & Toe slab & Heel slab & \multicolumn{2}{|l|}{} \\
\cline { 3 - 6 } & Ast. mm2 & Bar Dia. \& Spacing & Ast. mm2 & Bar Dia. \& Spacing \\
\hline 6 & 400 & 505.63 & $\begin{array}{l}\phi 12 \\
@ 150 \mathrm{~mm}\end{array}$ & 741.68 & $\begin{array}{l}\Phi 16 \\
@ 150 \mathrm{~mm}\end{array}$ \\
\hline
\end{tabular}




\begin{tabular}{|l|l|l|l|l|l|}
\hline 8 & 650 & 887.99 & $\begin{array}{l}\Phi 20 \\
@ 150 \mathrm{~mm}\end{array}$ & 3623.94 & $\phi 25 \quad @ 135 \mathrm{~mm}$ \\
\hline 10 & 750 & 2217.81 & $\begin{array}{l}\Phi 25 \\
@ 150 \mathrm{~mm}\end{array}$ & 3854.36 & $\Phi 32 \quad @ 150 \mathrm{~mm}$ \\
\hline 12 & 800 & 2343.51 & $\begin{array}{l}\Phi 25 \\
@ 150 \mathrm{~mm}\end{array}$ & 4069.80 & $\begin{array}{l}\Phi 25 \\
@ 150 \mathrm{~mm}\end{array}$ \\
\hline 15 & 900 & 3130.30 & $\begin{array}{l}\Phi 32 \\
@ 150 \mathrm{~mm}\end{array}$ & 5079.49 & $\begin{array}{l}\Phi 36 \\
@ 150 \mathrm{~mm}\end{array}$ \\
\hline
\end{tabular}

\begin{tabular}{|c|c|c|c|c|c|}
\hline $\begin{array}{l}\text { Ast. } \\
\mathrm{mm} 2\end{array}$ & 960 & 1560 & 1800 & 1920 & 2160 \\
\hline $\begin{array}{l}\text { Bar Dia. } \\
\& \\
\text { Spacing }\end{array}$ & $\begin{array}{l}\Phi 10 \\
@ 80 \\
\mathrm{~mm}\end{array}$ & $\begin{array}{l}\Phi 12 \\
@ 75 \\
\mathrm{~mm}\end{array}$ & $\begin{array}{l}\Phi 16 \\
@ 100 \mathrm{~m} \\
\mathrm{~m}\end{array}$ & $\begin{array}{l}\Phi 16 \\
@ 100 \\
\text { mm }\end{array}$ & $\begin{array}{l}\text { Ф16 } \\
@ 90 \mathrm{~mm}\end{array}$ \\
\hline
\end{tabular}

The R.C.C. steps / plates projecting in backfill are main key elements in this type of wall. The Resisting force developed due to these steps is function of depth of these steps below top of wall, surface roughness of concrete plates, degree of compaction of backfill and specific weight of backfill. The steps are developing frictional force due to their anchorage in backfill and steps are reinforced with sufficient steel required for tensile stress developed in it due to pulling effect. Though these steps are standing as free cantilever in backfill, they will not be designed as cantilever as it is assumed as backfill is compacted.

The details of forces acting and design of these concrete steps is as shown in Table 14

Table 14: Concrete Step analysis and design details

\begin{tabular}{|c|c|c|c|c|}
\hline \multirow{2}{*}{$\begin{array}{l}\text { Ht. of wall } \\
\mathrm{m}\end{array}$} & \multicolumn{2}{|c|}{$\begin{array}{l}\text { Step } \\
\text { Dimensions }\end{array}$} & \multicolumn{2}{|l|}{ Location } \\
\hline & Width & Depth & $\begin{array}{l}\text { Depth below } \\
\text { Top }\end{array}$ & $\begin{array}{l}\text { In Fill } \\
\text { Embedment }\end{array}$ \\
\hline 6 & 0.4 & 0.3 & 4.0 & 3.5 \\
\hline 8 & 0.5 & 0.3 & 5.5 & 4.5 \\
\hline 10 & 0.6 & 0.3 & 6.5 & 5.5 \\
\hline 12 & 0.65 & 0.4 & 7.5 & 6.5 \\
\hline 15 & 0.7 & 0.45 & 9.5 & 7.5 \\
\hline
\end{tabular}

\begin{tabular}{|l|l|l|l|}
\hline \multicolumn{2}{|l|}{$\begin{array}{l}\text { Reinforcement } \\
\text { Details }\end{array}$} & $\begin{array}{l}\text { Step spacing along } \\
\text { length }\end{array}$ & $\begin{array}{l}\text { Frictional } \\
\text { force } \\
\text { developed }\end{array}$ \\
\hline Dia & No & 2.0 & 67.68 \\
\hline 12 & 4 & 2.0 & 151.47 \\
\hline 12 & 6 & 2.0 & 244.30 \\
\hline 12 & 8 & 1.5 & 342.23 \\
\hline 12 & 12 & 1.25 & 517.10 \\
\hline 16 & 10 & & \\
\hline
\end{tabular}

In this type of wall the nature of moment variation will be similar as that of Cantilever retaining wall but there will be drastic change in moment at the point where concrete step is projected inside backfill. Up to this point the moment will be function of height of backfill but below this the moment will be algebraic sum of both resisting and destructive moments i.e. Destructive moment due to backfill and resisting moment of frictional force developed due to step.

The steel reinforcement will be provided not only adhering to moment values but with also consideration to minimum steel quantities and Practical site considerations also.

The table 15 and 16 shows the moment variation and steel reinforcement provided for this stepped cantilever wall 
Table 15: Moment Variation Along length of stem for Stepped cantilever Wall

\begin{tabular}{|c|c|c|c|c|c|c|}
\hline & \multicolumn{2}{|l|}{$6 \mathrm{~m}$} & \multicolumn{2}{|l|}{$8 \mathrm{~m}$} & \multicolumn{2}{|l|}{$10 \mathrm{~m}$} \\
\hline & $\begin{array}{l}\text { Moment } \\
\mathrm{KNm}\end{array}$ & $\begin{array}{l}\text { D Prod } \\
\text { Mm }\end{array}$ & $\begin{array}{l}\text { Moment } \\
\text { KNm }\end{array}$ & $\begin{array}{l}\text { D Prod } \\
\mathrm{mm}\end{array}$ & $\begin{array}{l}\text { Moment } \\
\mathrm{KNm}\end{array}$ & $\begin{array}{l}\text { D Prod } \\
\text { mm }\end{array}$ \\
\hline $0-\mathrm{L} / 4$ & 3.375 & 225 & 8.0 & 250 & 15.63 & 300 \\
\hline $\mathrm{L} / 4-\mathrm{L} / 2$ & 27.0 & 250 & 64.0 & 300 & 125 & 400 \\
\hline $\mathrm{L} / 2-2 \mathrm{~L} / 3$ & 91.12 & 275 & 216.0 & 450 & 421.87 & 600 \\
\hline $2 \mathrm{~L} / 3-\mathrm{L}$ & 35.52 & 300 & 310.04 & 550 & 674.27 & 750 \\
\hline
\end{tabular}

\begin{tabular}{|l|l|l|l|l|}
\hline & $12 \mathrm{~m}$ & $15 \mathrm{~m}$ & \multicolumn{2}{|l|}{$\begin{array}{l}\text { D Prod } \\
\text { Mm }\end{array}$} \\
\cline { 2 - 5 } & $\begin{array}{l}\text { Moment } \\
\text { KNm }\end{array}$ & $\begin{array}{l}\text { Moment } \\
\text { KNm }\end{array}$ & 52.7 & 400 \\
\hline L-L/4-L/2 & 27.0 & 350 & 421.9 & 700 \\
\hline L/2-2L/3 & 216.0 & 500 & 1423.8 & 1000 \\
\hline 2L/3-L & 729 & 750 & 2944.08 & 1400 \\
\hline
\end{tabular}

Table 16: Reinforcement details along Height of stem

\begin{tabular}{|c|c|c|c|c|c|c|}
\hline \multirow{3}{*}{$\begin{array}{l}\text { Ht. of } \\
\text { wall } \\
\mathrm{m} .\end{array}$} & \multirow{3}{*}{$\begin{array}{l}\text { Moment Variation along } \\
\text { length of stem }(\mathrm{KNm})\end{array}$} & \multicolumn{5}{|c|}{ Steel prov. In Vertical wall } \\
\hline & & \multicolumn{2}{|c|}{ Stem Thickness } & & \multirow{2}{*}{\multicolumn{2}{|c|}{ Bar Dia. \& Spacing }} \\
\hline & & Dreq. $\mathrm{Mm}$ & Dprov. $\mathrm{Mm}$ & Ast $\mathrm{mm} 2$ & & \\
\hline 6 & 35.52 & 138.94 & 300 & 500.82 & $\Phi 12$ & \\
\hline 8 & 310.04 & 410.49 & 500 & 2732.36 & $\Phi 20$ & @ $115 \mathrm{~mm}$ \\
\hline 10 & 674.27 & 605.35 & 700 & 4274.66 & $\Phi 25$ & $@ 115 \mathrm{~mm}$ \\
\hline 12 & 1385.77 & 867.83 & 950 & 4238.38 & $\Phi 25$ & @ 115mm \\
\hline 15 & 2944.08 & 1264.93 & 1350 & 9803.36 & Ф32 & @80mm \\
\hline
\end{tabular}

\subsection{Unit Cost per Meter of Wall}

\section{A) Counter fort Retaining Wall:}

The cost of counter fort retaining wall includes cost of concrete for stem, counter fort and base slab is added, and the steel quantity is calculated from actual steel used with some provision for wastage also. For counter fort retaining wall, the cost of wall is calculated for total spacing of counter forts and from this per meter cost of wall is calculated.

The cost per running meter for counter fort retaining wall for various retain heights is as shown in table

Table 17: Cost per Running Meter for Counter fort Retaining

\begin{tabular}{|c|c|c|c|c|}
\hline Ht. of wall & \multicolumn{2}{|l|}{$6 \mathrm{~m}$} & \multicolumn{2}{|l|}{$8 \mathrm{~m}$} \\
\hline Location & Concretem 3 & Steel kg & Concretem3 & Steel kg \\
\hline Stem & 1.2 & 76.08 & 2.4 & 137.6 \\
\hline Base slab & 0.98 & 66.16 & 1.49 & 80.08 \\
\hline $\begin{array}{l}\text { Counter } \\
\text { Forts }\end{array}$ & 2.7 & 137.2 & 5.18 & 234.05 \\
\hline Total & 4.88 & 279.44 & 9.07 & 451.73 \\
\hline Rate & 3500 & 43 & 3500 & 43 \\
\hline Amount & 17080 & 12015.9 & 31745 & 19424.39 \\
\hline Sum & \multicolumn{2}{|l|}{29095.9} & \multicolumn{2}{|l|}{51169.4} \\
\hline & \multicolumn{2}{|l|}{29100} & \multicolumn{2}{|l|}{51170} \\
\hline
\end{tabular}




\begin{tabular}{|l|l|l|l|l|l|}
\hline $10 \mathrm{~m}$ & \multicolumn{2}{|l|}{$12 \mathrm{~m}$} & $15 \mathrm{~m}$ & \\
\hline Concretem3 & Steel kg & Concretem3 & Steel kg & Concretem3 & Steel kg \\
\hline 3.5 & 156.8 & 5.4 & 251.52 & 8.25 & 439.7 \\
\hline 2.52 & 139.86 & 3.9 & 229.82 & 7.7 & 475.28 \\
\hline 8.5 & 527.98 & 16.34 & 765.5 & 27.64 & 1810.55 \\
\hline 14.52 & 824.64 & 25.64 & 1246.84 & 43.59 & 2725.53 \\
\hline 3500 & 43 & 3500 & 43 & 3500 & 43 \\
\hline 50820 & 35459.52 & 89740 & 53614.12 & 152565 & 117197.79 \\
\hline 86279.52 & & 143354.1 & & 269763 & \\
\hline 86280 & & 143360 & & 269770 & \\
\hline
\end{tabular}

\section{B) Stepped Cantilever Retaining Wall:}

As like for counter fort retaining wall, the cost of stepped cantilever retaining wall will be calculated firstly as per spacing of steps in backfill along length of wall and hence it is transferred to per meter cost. The construction practice for stepped cantilever wall will not be very special than cantilever wall hence except extra amount for backfill compaction, no any extra provision is made in cost calculation.

Table 18: Cost per running meter for Stepped Cantilever Retaining Wall

\begin{tabular}{|c|c|c|c|c|c|c|}
\hline \multirow{2}{*}{\begin{tabular}{|l|}
$\begin{array}{l}\text { Ht. of } \\
\text { wall }\end{array}$ \\
Location \\
\end{tabular}} & \multicolumn{3}{|c|}{$6 \mathrm{~m}$} & \multicolumn{3}{|l|}{$8 \mathrm{~m}$} \\
\hline & \multicolumn{2}{|c|}{ Concretem 3} & Steel kg & \multicolumn{2}{|c|}{ Concretem 3} & Steel kg \\
\hline Stem & \multicolumn{2}{|l|}{3} & 142.78 & \multicolumn{2}{|l|}{4.8} & 476.72 \\
\hline Base slab & \multicolumn{2}{|c|}{2.28} & 84.91 & \multicolumn{2}{|l|}{4.2} & 370.71 \\
\hline Steps & \multicolumn{2}{|c|}{0.25} & 8.2 & \multicolumn{2}{|l|}{0.39} & 16.63 \\
\hline Total & \multicolumn{2}{|c|}{5.53} & 235.89 & \multicolumn{2}{|l|}{9.39} & 864.06 \\
\hline Rate & \multicolumn{2}{|c|}{3500} & 43 & \multicolumn{2}{|l|}{3500} & 43 \\
\hline Amount & \multicolumn{2}{|c|}{19355} & 10143.3 & \multicolumn{2}{|l|}{32865} & 37154.58 \\
\hline \multirow[t]{2}{*}{ Sum } & \multicolumn{3}{|c|}{29498.3} & \multicolumn{3}{|c|}{70019.58} \\
\hline & \multicolumn{3}{|c|}{29500} & \multicolumn{3}{|l|}{70000} \\
\hline \multicolumn{3}{|l|}{$10 \mathrm{~m}$} & \multicolumn{2}{|l|}{$12 \mathrm{~m}$} & \multicolumn{2}{|l|}{$15 \mathrm{~m}$} \\
\hline \multicolumn{2}{|c|}{ Concretem 3} & Steel kg & Concretem3 & $\begin{array}{l}\text { Steel } \\
\mathrm{kg}\end{array}$ & Concretem3 & Steel $\mathrm{kg}$ \\
\hline \multicolumn{2}{|l|}{8.6} & 972.2 & 11.52 & 602.65 & 25.5 & 1688.23 \\
\hline \multicolumn{2}{|l|}{7.8} & 623.21 & 8.29 & 500 & 11.8 & 850.07 \\
\hline \multicolumn{2}{|l|}{0.55} & 26.18 & 0.9 & 59.69 & 1.2 & 100.88 \\
\hline \multicolumn{2}{|l|}{16.95} & 1621.59 & 20.71 & 1162.3 & 38.5 & 2639.18 \\
\hline \multicolumn{2}{|l|}{3500} & 43 & 3500 & 43 & 3500 & 43 \\
\hline \multicolumn{2}{|l|}{59325} & 69728.4 & 72485 & 49981 & 134750 & 113485 \\
\hline \multicolumn{3}{|l|}{129053} & \multicolumn{2}{|l|}{122466} & \multicolumn{2}{|l|}{248235} \\
\hline \multicolumn{3}{|l|}{129050} & 122470 & & 248240 & \\
\hline
\end{tabular}

\subsection{Cost Comparison:}

The cost per meter for all these three proposed types is tabulated above. In table 6.19 the comparison of concrete quantity per meter for different wall heights and different wall types are shown. 
Table 19: Comparison of Concrete for Different Walls

\begin{tabular}{|l|l|l|}
\hline $\begin{array}{l}\text { Wall Ht. } \\
\mathrm{m}\end{array}$ & $\begin{array}{l}\text { Counter } \\
\text { fort wall }\end{array}$ & $\begin{array}{l}\text { Stepped } \\
\text { Cantilever } \\
\text { wall }\end{array}$ \\
\hline 6 & 4.88 & 5.53 \\
\hline 8 & 9.07 & 9.39 \\
\hline 10 & 14.52 & 16.95 \\
\hline 12 & 25.64 & 20.72 \\
\hline 15 & 43.59 & 38.5 \\
\hline
\end{tabular}

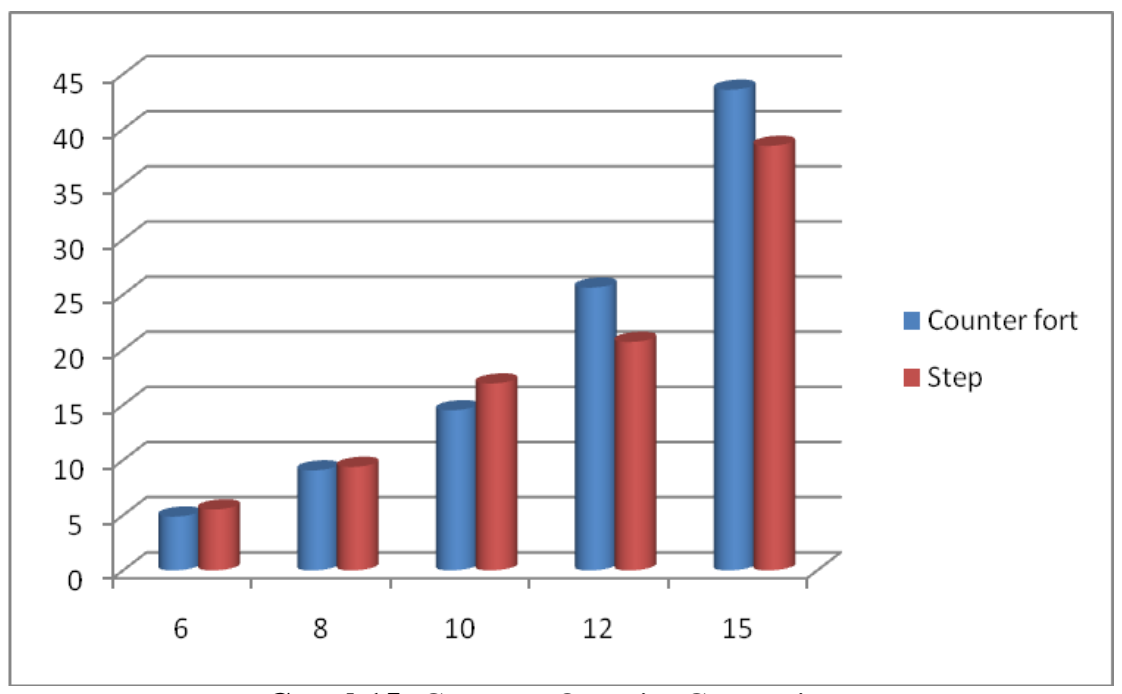

Graph15: Concrete Quantity Comparison

Table 20: Steel reinforcement per meter of wall

\begin{tabular}{|l|l|l|}
\hline $\begin{array}{l}\text { Wall Ht. } \\
\mathrm{m}\end{array}$ & Counter fort wall & $\begin{array}{l}\text { Stepped Cantilever } \\
\text { wall }\end{array}$ \\
\hline 6 & 279.44 & 235.89 \\
\hline 8 & 451.73 & 864.96 \\
\hline 10 & 824.64 & 1621.59 \\
\hline 12 & 1246.84 & 1162.34 \\
\hline 15 & 2725.53 & 2639.18 \\
\hline
\end{tabular}

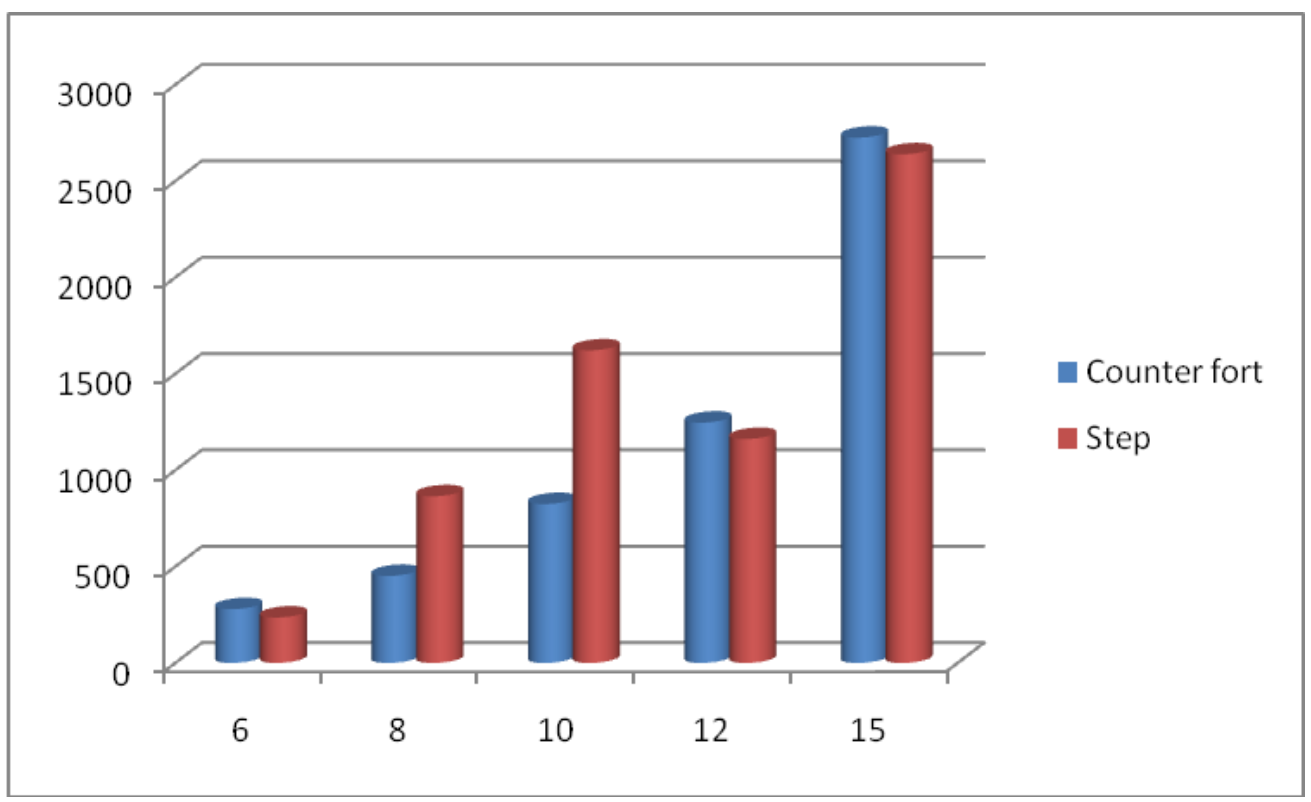

Graph 16: Reinforcement Quantity Comparison 
The table 21 shows final cost comparison of all these wall types for same heights and graph 17 showing variation.

Table 21: Final Cost Comparison

\begin{tabular}{|l|l|l|}
\hline $\begin{array}{l}\text { Wall Ht. } \\
\mathrm{m}\end{array}$ & $\begin{array}{l}\text { Counter fort } \\
\text { wall }\end{array}$ & $\begin{array}{l}\text { Stepped } \\
\text { Cantilever wall }\end{array}$ \\
\hline 6 & 29100 & 29500 \\
\hline 8 & 51170 & 70000 \\
\hline 10 & 86280 & 129050 \\
\hline 12 & 143360 & 122470 \\
\hline 15 & 269770 & 248240 \\
\hline
\end{tabular}

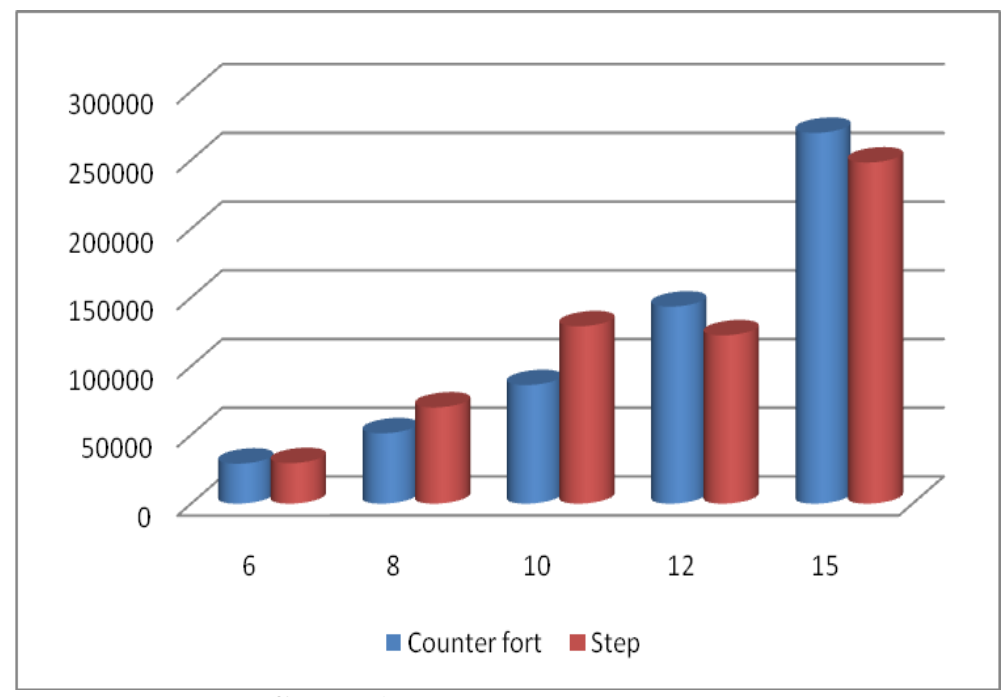

Graph 17: Final Cost Comparison

It is clear from table that for heights from $8.0 \mathrm{M}$ to $10.0 \mathrm{M}$ counter fort retaining wall is giving economical results. Hence counter fort wall is better alternative for retaining heights up to $10.0 \mathrm{M}$. Other wall types may also be checked depending on actual site conditions.

The stepped cantilever is giving best result for height more than $10.0 \mathrm{M}$, from this height counter fort retaining walls are being uneconomical.

\section{CONCLUSION}

Cantilever retaining walls are economically suited for wall heights up to $6.0 \mathrm{M}$ and hence for height up to $6.0 \mathrm{M}$, no other alternative is necessary.

Counter fort retaining walls are suitable for retaining wall heights $8.0 \mathrm{M}$ to $10.0 \mathrm{M}$ for standard site conditions assumed. The other types of wall may also be tried for different site conditions.

At first instant, Stepped cantilever Retaining wall are economically best suited for wall heights from $11.0 \mathrm{M}$ to 15.0 M. this is proving to be better alternative for large wall heights as more than 11.0 M. Its mechanism is proven and used in many civil engineering structures.

\section{REFERENCES}

[1]. S.K Bhatia and R.M Baker, "Difference between Cantilever and Gravity retaining walls under static conditions", Indian Geotechnical Journal, Vol.15, No.3, May 1985.

[2]. Kaare Hoeg and Ramesh Murarka, "Probabilistic Analysis and Design of a Retaining Wall", Journal of Geotechnical Engineering Division, Vol.100, March 1994.

[3]. Swami Saran, "Displacement Dependent Earth Pressure in Retaining Walls", Indian Geotechnical Journal, Indian Geotechnical Journal, Vol.20, July 1990.

[4]. Leo Cassagrande, "Comments on Conventional Design of Retaining Structures", Journal of the soil mechanics and foundation division, ASCE, Vol.99, Feb2003.

\section{BIOGRAPHIES}

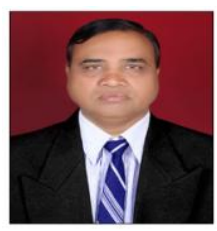

Prof. Dr.Patil S.S., B.E(Civil), M.E (Civil -Structures), Ph.D (B.U., Bangalore), Chairman Indian Society of Structural Engineers Solapur Local Centre, Professor \& Head of Civil Engineering Department, Walchand Institute of Technology, Ashok Chowk Solapur. (M.S) INDIA.

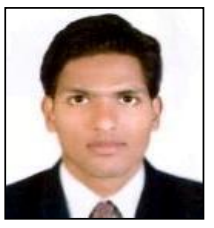

Mr. Bagban Aamir A.R, B.E (Civil), M.E (Civil - Structures), M.E Student of WIT Solapur (M.S) INDIA 